COMMUNICATIONS IN

ANALYSIS AND GEOMETRY

Volume 12, Number 1, 59-91, 2004

\title{
On the Asymptotic Scalar Curvature Ratio of Complete Type I-like Ancient Solutions to the Ricci Flow on Noncompact 3-manifolds
}

\author{
Bennett Chow and Peng Lu
}

\section{Introduction.}

Complete noncompact Riemannian manifolds with nonnegative sectional curvature arise naturally in the Ricci flow when one takes the limits of dilations about a singularity of a solution of the Ricci flow on a compact 3-manifold [H-95a]. To analyze the singularities in the Ricci flow one needs to understand these manifolds in depth. There are three invariants, asymptotic scalar curvature ratio, asymptotic volume ratio and aperture, that have been used to study the geometry of these manifolds at infinity.

Let $\left(\mathcal{M}^{n}, g\right)$ be a complete noncompact Riemannian manifold with nonnegative sectional curvature and let $O \in \mathcal{M}$ be some point which we call the origin. The asymptotic scalar curvature ratio (ASCR) is defined by

$$
\operatorname{ASCR}(\mathcal{M}, g)=\limsup _{d(x, O) \rightarrow \infty} R(x) \cdot d(x, O)^{2},
$$

where $R$ is the scalar curvature. ASCR is a measure of how non-flat the manifold is at infinity. Since the sectional curvature is nonnegative, there is a positive constant $c$ depending only on $n$ such that

$$
\begin{aligned}
c^{-1} \cdot \limsup _{d(x, O) \rightarrow \infty}|R m(x)| \cdot d(x, O)^{2} & \leq \operatorname{ASCR}(\mathcal{M}, g) \\
& \leq c \cdot \limsup _{d(x, O) \rightarrow \infty}|R m(x)| \cdot d(x, O)^{2}
\end{aligned}
$$

where $R m$ is the Riemann curvature tensor. In the literature sometimes

$$
\limsup _{d(x, O) \rightarrow \infty}|R m(x)| \cdot d(x, O)^{2}
$$

is used as the definition of ASCR. It is clear that ASCR is independent of the choice of origins and is invariant under scaling. 
ASCR has been used to study gap theorems. In particular, in [ESS-89] Eschenburg, Schroeder and Strake proved that if $\left(\mathcal{M}^{2 k+1}, g\right)$ is a complete noncompact odd-dimensional Riemannian manifold with positive sectional curvature, then $\operatorname{ASCR}(\mathcal{M}, g)>0$. These types of results are generally referred to as gap theorems since they show the existence of a gap between flat $\mathbb{R}^{n}$ and metrics of positive curvature on $\mathbb{R}^{n}$. Gap theorems have been proved by Greene-Wu [GW-82], Kasue and Sugahara [KS-87], and Drees [D-94]. For a survey of the history of gap theorems related to the notion of ASCR, see [G-97]. ASCR has also been used to study the structure of manifolds at infinity [PT-01].

Below we give a few examples about how ASCR is used in singularity analysis in the Ricci flow.

1. In [H-95a] Hamilton showed that for a solution to the Ricci flow on a compact 3-manifold forming a Type II singularity and satisfying an injectivity radius estimate, there exists a sequence of dilations converging to a complete solution $\left(\mathcal{M}_{\infty}^{3}, g_{\infty}(t)\right)$ defined for all $t \in(-\infty, \infty)$ with nonnegative bounded sectional curvature and attaining its maximum of the scalar curvature on space and time. In [H-93b] it is shown using the differential Harnack inequality of Li-Yau-Hamilton type [H-93a] that the universal covering solution $\left(\widetilde{\mathcal{M}}_{\infty}^{3}, \tilde{g}_{\infty}(t)\right)$ of such a solution must be a stationary solution (also called Ricci solitons) of the Ricci flow in the space of metrics modulo diffeomorphisms flowing along a gradient vector field, that is, there exists a 1-parameter family of diffeomorphisms $\varphi_{t}: \widetilde{\mathcal{M}}_{\infty}^{3} \rightarrow \widetilde{\mathcal{M}}_{\infty}^{3}$ such that $\tilde{g}_{\infty}(t)=\varphi_{t}^{*}\left(\tilde{g}_{\infty}(0)\right)$ and the 1-parameter family of vector fields $X(t)$ generated by $\varphi_{t}$ are the gradients of functions $f(t)$. In $\S 20$ of [H-95a] it is shown that such a Ricci soliton must have $\operatorname{ASCR}(g(t))=\infty$ for all $t \in(-\infty, \infty)$.

2. In $\S 22$ of [H-95a] Hamilton showed using a geometric result about bumps of curvature from $\S 21$ of [H-95a] that if a complete solution to the Ricci flow with bounded curvature has $\operatorname{ASCR}(g(t))=\infty$, then one can perform dimension reduction. In particular, provided there is a local injectivity radius estimate, there exists a sequence of points $\left\{y_{\alpha}\right\}_{\alpha \in \mathbb{N}}$ in $\mathcal{M}^{3}$ with $\lim _{\alpha \rightarrow \infty} d_{g(0)}\left(y_{\alpha}, O\right)=\infty$ such that the sequence of dilated solutions on balls $\left\{\left(B_{g_{\alpha}(0)}\left(y_{\alpha}, r_{\alpha}\right), g_{\alpha}(t)\right), y_{\alpha}\right\}_{\alpha \in \mathbb{N}}$, where

$$
g_{\alpha}(t)=R\left(g_{\alpha}\right)\left(y_{\alpha}, 0\right) g\left(t \cdot R\left(g_{\alpha}\right)^{-1}\left(y_{\alpha}, 0\right)\right)
$$

and $\lim _{\alpha \rightarrow \infty} r_{\alpha}^{2} R\left(g_{\alpha}\right)\left(y_{\alpha}, 0\right)=\infty$, converges to complete solution to the Ricci flow $\left(\mathcal{P}_{\infty}, k_{\infty}(t), y_{\infty}\right)$ with bounded nonnegative sectional 
curvature which splits metrically as the product of $\mathbb{R}$ and a solution on a surface with positive curvature. This is why it is important to know when a noncompact ancient solution has infinite asymptotic scalar curvature ratio, when this is true one should be able to perform dimension reduction.

3. In $\S 5.3$ of [H-97] it is shown that for an ancient solution $(\mathcal{M}, g(t))$ to the Ricci flow which is complete with bounded positive curvature operator and satisfies certain pinching conditions, then $\operatorname{ASCR}(\mathcal{M}, g(t))=\infty$ if and only if for some fixed time the solution metric has an arbitrarily necklike end (see $\S 2$ below for a definition).

The asymptotic volume ratio is defined by

$$
A V R(g) \doteqdot \lim _{r \rightarrow \infty} \frac{\operatorname{Vol}[B(O, r)]}{r^{n}}
$$

where $B(O, r)=\{x: d(O, x)<r\}$. It is also used in singularity analysis in the Ricci flow. In particular, in $\S 19$ of [H-95a] Hamilton showed that a complete ancient Type I-like solution to the Ricci flow with bounded positive curvature operator and finite $\operatorname{ASCR}$ must have $\operatorname{AVR}(g(t))>0$ for all $t$. He also showed there that the scalar curvature decays exactly quadratically:

$$
0<c \leq R(x, t) d_{g(t)}(x, O)^{2} \leq C
$$

where $c$ and $C$ depend on $O$ and $t$.

This paper arises from exploring the following idea inspired by the work of Hamilton [H-97]. If a piece of a positively curved complete noncompact manifold is sufficiently close to a long standard cylinder, then its asymptotic scalar curvature ratio is large.

\section{Main results.}

To state the main result of this paper we need to define the so-called $(\varepsilon, k, L)$ necks. First we recall a few basic definitions concerning necks from $§ 3.2$ of [H-97]. A topological neck in a differentiable manifold $\mathcal{M}^{n}$ is a local diffeomorphism

$$
N: S^{n-1} \times[a, b] \rightarrow \mathcal{M}^{n}
$$

for some $a<b$.

Let $g$ be a Riemannian metric on $\mathcal{M}$ and $g \doteqdot N^{*}(g)$ be the pulled-back metric on on $S^{n-1} \times[a, b]$. A neck is called normal if it satisfies the following five conditions. 
1. (good slices) Each slice $S^{n-1} \times\{z\} \subset S^{n-1} \times[a, b], z \in[a, b]$, has constant mean curvature with respect to $g$.

2. (good parametrizations of the slices) The identity map

$$
\iota:\left(S^{n-1} \times\{z\}, \bar{g}\right) \rightarrow\left(S^{n-1} \times\{z\}, g\right)
$$

is harmonic for all $z \in[a, b]$, where $\bar{g}$ is the standard metric.

3. (taking in account that conformal maps of $S^{2}$ are harmonic) When $n=3$ the center of mass of $S^{2} \times\{z\} \subset \mathbb{R}^{3} \times\{z\}$ with respect to $g$ is the origin:

$$
\int_{S^{2} \times\{z\}} \vec{x} d A_{g}(\vec{x})=\overrightarrow{0} \in \mathbb{R}^{3}
$$

for all $z \in[a, b]$, where $d A_{g}$ is the area form.

4. (good spacing of the slices) The spacing of the slices are normalized using volume by

$$
\operatorname{Vol}\left(S^{n-1} \times[z, w], g\right)=\omega_{n-1} \int_{z}^{w} r(y)^{n} d y
$$

where

$$
r(y) \doteqdot\left(\frac{\operatorname{Area}\left(S^{n-1} \times\{y\}, g\right)}{\omega_{n-1}}\right)^{\frac{1}{n-1}}
$$

is called the mean radius.

5. (aligning the parametrizations) If $\bar{V}$ is a Killing vector field on a slice $\left(S^{n-1} \times\{z\}, \bar{g}\right)$, then the unit normal vector field $\nu$ of $S^{2} \times\{z\} \subset$ $\mathbb{R}^{3} \times\{z\}$ with respect to $g$ satisfies

$$
\int_{S^{n-1} \times\{z\}} \bar{g}(\bar{V}, \nu) d A_{\bar{g}}=0
$$

for all $z \in[a, b]$.

Note that in condition 4 it is important that the power of $r(y)$ is $n$, not $n-1$.

A neck is called $(\varepsilon, k)$-cylindrical if 
1. (conformally close to cylindrical in $C^{0}$ ) the metric

$$
\widehat{g}(\theta, z) \doteqdot r(z)^{-2} g(\theta, z)
$$

satisfies

$$
|\widehat{g}-\bar{g}|_{\bar{g}} \leq \varepsilon,
$$

2. (conformally close to cylindrical up to $C^{k}$ )

$$
\left|\bar{\nabla}^{j} \widehat{g}\right|_{\bar{g}} \leq \varepsilon
$$

for all $1 \leq j \leq k$, where $\bar{\nabla}$ is the covariant derivative with respect to $\bar{g}$

\section{3. (mean radius changes slowly)}

$$
\left|\frac{d^{j} \log r(z)}{d z^{j}}\right| \leq \varepsilon
$$

for all $1 \leq j \leq k$.

A neck $N$ in a manifold $\mathcal{M}^{n}$ is called an embedded neck if $N: S^{n-1} \times$ $[a, b] \rightarrow \mathcal{M}^{n}$ is an embedding.

Definition 1. (i) A map $N: S^{n-1} \times[a, b] \rightarrow \mathcal{M}$ is called an $(\varepsilon, k, L)$-neck if it is a normal $(\varepsilon, k)$-cylindrical neck and $b-a \geq 2 L$.

(ii) A complete Riemannian manifold $(\mathcal{M}, g)$ with exactly one topological end is called to have an arbitrarily necklike end if for every $(\varepsilon, k, L)$ there exists an $(\varepsilon, k, L)$-neck in $(\mathcal{M}, g)$.

Definition (ii) is from $\S 5.3$ of [H-97] (p. 71). We write $\left(\varepsilon_{1}, k_{1}, L_{1}\right) \preccurlyeq$ $\left(\varepsilon_{2}, k_{2}, L_{2}\right)$ if $\varepsilon_{1} \leq \varepsilon_{2}, k_{1} \geq k_{2}$ and $L_{1} \geq L_{2}$. In the next lemma we collect a few simple properties of $(\varepsilon, k, L)$-necks.

Lemma 2. (i) $(\varepsilon, k, L)$-necks are scale-invariant, i.e., if $N: S^{n-1} \times[a, b] \rightarrow$ $\left(M^{n}, g\right)$ is an $(\varepsilon, k, L)$-neck then $N: S^{n-1} \times[a, b] \rightarrow\left(M^{n}, \lambda^{2} \cdot g\right)$ is also an $(\varepsilon, k, L)$-neck for any $\lambda>0$.

(ii) If $N: S^{n-1} \times[a, b] \rightarrow \mathcal{M}$ is an $\left(\varepsilon_{1}, k_{1}, L_{1}\right)$-neck and $\left(\varepsilon_{1}, k_{1}, L_{1}\right) \preccurlyeq$ $\left(\varepsilon_{2}, k_{2}, L_{2}\right)$, then $N$ is an $\left(\varepsilon_{2}, k_{2}, L_{2}\right)$-neck.

(iii) If $S_{r}^{n-1}$ is the sphere of radius $r$, then for all $\varepsilon>0$ and $k \in \mathbb{N}$ the product manifold $\mathcal{M}=S_{r}^{n-1} \times[-r L, r L]$ has an $(\varepsilon, k, L)$-neck given by the obvious map. 
The main result of this paper is the following theorem which will be proved in $\S 4$.

Theorem 3. For every odd integer $n \geq 3$ and every positive $C_{0}<\infty$ there exists $\left(\varepsilon_{0}, k_{0}, L_{0}\right)$ such that if $\left(\mathcal{M}^{n}, g\right)$ is a complete, orientable, noncompact Riemannian manifold with bounded positive sectional curvature and $\mathcal{M}$ contains an embedded $(\varepsilon, k, L)$-neck with $(\varepsilon, k, L) \preccurlyeq\left(\varepsilon_{0}, k_{0}, L_{0}\right)$, then

$$
\operatorname{ASCR}(g) \geq C_{0} .
$$

The main result may be thought of as a odd-dimensional quantitative version of the "only if" part of Theorem 3.1 in $\S 5.3$ of [H-97], which states that a complete noncompact ancient solution to the Ricci flow on a four-manifold with bounded positive curvature operator satisfying certain pinching conditions has an arbitrarily necklike end at sometime if and only if the asymptotic scalar curvature ratio is infinite. In particular, we obtain a weaker characterization of $\operatorname{ASCR}(g(t))=\infty$, which is one of the ingredients used in the proof of Theorem 4 below.

The main result has the following useful consequence in dimension three. Recall that an ancient solution is called Type-I like if

$$
\sup _{\mathcal{M}^{n} \times(-\infty, 0]}|t| \cdot|R m(x, t)|<\infty .
$$

Theorem 4. If $\left(\mathcal{M}^{3}, g(t)\right),-\infty<t<\omega$, is a complete noncompact ancient Type I-like solution to the Ricci flow with bounded positive sectional curvature on an orientable 3-manifold, then $\operatorname{ASCR}(g(t))=\infty$ for all $t \in(-\infty, \omega)$.

We shall prove this theorem in $\S 5$. This answers a conjecture of Hamilton when $n=3$. In $\S 22$ of [H-95a] (p. 93) he writes:

We do not know any examples of complete noncompact ancient solutions of positive curvature operator with $R s^{2}<\infty$ and $R|t|<\infty$, and we conjecture none exist, since the curvature has had plenty of space and time to dissipate.

Conjecture 5. There does not exist complete noncompact ancient Type I-like solutions to the Ricci flow with bounded positive curvature operator.

By the theorem above such solutions, if they were to exist, must necessarily have infinite asymptotic scalar curvature ratio. When $n=2$ it is proved in Theorem 26.1 of [H-95a] that there does not exist complete noncompact ancient Type I-like solutions to the Ricci flow with positive sectional curvature. 
For the mean curvature flow, which in general has very similar properties as the Ricci flow, there are no complete noncompact strictly convex Type I-like ancient solutions by Huisken's classification using his monotonicity formula [Hu-90].

\section{Relative volumes and necks.}

The main result of this section, Proposition 10 below, is to combine the relative volume comparison theorem (Lemma 12) and the existence of an embedded $(\varepsilon, k, L)$-neck to show that there are small relative volumes. Note that this result holds in both odd and even dimensions.

\subsection{Busemann functions.}

Let $\left(\mathcal{M}^{n}, g\right)$ be a complete noncompact Riemannian manifold. Given a point $Q \in \mathcal{M}$ and a ray $\gamma$ emanating from $Q$, the Busemann function $b_{\gamma}: M \rightarrow \mathbb{R}$ associated to $\gamma$ is defined by

$$
b_{\gamma}(x) \doteqdot \lim _{t \rightarrow+\infty}[t-d(\gamma(t), x)] .
$$

Let $\mathcal{R}$ be the set of all rays emanating from $Q$. The Busemann function $b_{Q}: M \rightarrow \mathbb{R}$ with the base point $Q$ is defined by

$$
b_{Q}(x)=\sup _{\gamma \in \mathcal{R}} b_{\gamma}(x)
$$

We collect some well-known properties of Busemann functions on complete noncompact Riemannian manifolds with nonnegative sectional curvature in the following lemma (for a proof see, for example, [CG-72] and [LT-87]).

Lemma 6. Let $\left(\mathcal{M}^{n}, g\right)$ be a complete noncompact Riemannian manifold with nonnegative sectional curvature. Then

(i) The Busemann function $b_{Q}$ is proper, Lipschitz with Lipschitz constant 1 and bounded from below.

(ii) The sublevel sets $C_{r} \doteqdot\left\{x \in \mathcal{M}: b_{Q}(x) \leq r\right\}$ are compact and totally convex.

(iii) Let $S_{r} \doteqdot b_{Q}^{-1}(r)$ denote level set of the Busemann function. Then for any $r_{1} \leq r_{2}$

$$
S_{r_{1}}=\left\{x \in C_{r_{2}}: d\left(x, S_{r_{2}}\right)=r_{2}-r_{1}\right\} .
$$

(iv) $\left|b_{Q}(x)\right| \leq d(x, Q)$, which implies $B(Q, r) \subset C_{r}$. 
The following lemma says that any sufficiently long minimal geodesic segment emanating from $Q$ can be well approximated by a ray emanating from $Q$ and that the Busemann function is similar at infinity to the distance function to the base point $Q$ (see Li-Tam [LT-87] Theorem 2.3 on p. 177, Kasue [K-88] Lemma 1.4 on p. 598, Drees [D-94] Lemma 1 on p. 80 and for an exposition see $[\mathrm{CKE}])$. For the convenience of the reader we give a proof here.

Lemma 7. Let $\left(\mathcal{M}^{n}, g\right)$ be a complete noncompact Riemannian manifold with nonnegative sectional curvature. Define $\theta:[0, \infty) \rightarrow[0, \pi]$ by

$$
\theta(r)=\sup _{\sigma \in \mathcal{S}(r)} \inf _{\gamma \in \mathcal{R}} \measuredangle_{Q}\left(\sigma^{\prime}(0), \gamma^{\prime}(0)\right)
$$

where $\mathcal{S}(r)$ is the set of all minimal geodesic segments $\sigma$ of length $L(\sigma) \geq r$ emanating from $Q$. Then

(i) $\theta(r)$ is a nonincreasing function of $r$ and

$$
\lim _{r \rightarrow+\infty} \theta(r)=0 .
$$

(ii) The function $b_{Q}$ and $d(\cdot, Q)$ are asymptotically equal, more precisely

$$
(1-\theta[d(x, Q)]) \cdot d(x, Q) \leq b_{Q}(x) \leq d(x, Q)
$$

for all $x \in \mathcal{M}$.

Proof. (i) From the definition it is clear that $\theta(r)$ is a nonincreasing function of $r$. If $\lim _{r \rightarrow \infty} \theta(r) \neq 0$, there exists $\epsilon>0$, a sequence of points $p_{i} \in \mathcal{M}$ with $d\left(p_{i}, Q\right) \nearrow+\infty$, and minimal geodesic segments $\sigma_{i}$ joining $Q$ and $p_{i}$ parametrized by arc length such that

$$
\measuredangle_{Q}\left(\sigma_{i}^{\prime}(0), \gamma^{\prime}(0)\right) \geq \epsilon
$$

for each $i$ and all rays $\gamma \in \mathcal{R}$. By the compactness of the unit sphere in $T_{Q} \mathcal{M}$, there is a subsequence such that $\lim _{j \rightarrow+\infty} \sigma_{i_{j}}^{\prime}(0) \doteqdot V_{\infty}$ exists. Let $\sigma_{\infty}:[0,+\infty) \rightarrow \mathcal{M}$ be the unique geodesic with $\sigma_{\infty}(0)=Q$ and $\sigma_{\infty}^{\prime}(0)=$ $V_{\infty}$. It is clear that $\sigma_{\infty} \in \mathcal{R}$. In particular the condition

$$
\measuredangle_{Q}\left(\sigma_{i_{j}}^{\prime}(0), \sigma_{\infty}^{\prime}(0)\right) \geq \epsilon
$$

is impossible for large enough $j$. (i) is proved 
(ii) For any $x \in \mathcal{M}$ let $\sigma$ be a minimal geodesic from $Q$ to $x$ and $r=d(x, Q)$. Since $\mathcal{R}$ is a closed set, there is a ray $\gamma \in \mathcal{R}$ such that $\measuredangle_{Q}\left(\sigma^{\prime}(0), \gamma^{\prime}(0)\right) \leq \theta(r)$. Then it follows from sectional curvature $K_{\mathcal{M}} \geq 0$ and Toponogov's comparison theorem that $d(x, \gamma(r)) \leq \theta(r) \cdot r$. Hence

$$
b_{Q}(x) \geq b_{\gamma}(x) \geq r-d(x, \gamma(r)) \geq(1-\theta(r)) \cdot r .
$$

The lemma is proved.

\subsection{Necks in manifolds with positive sectional curvature.}

We call a neck $N: S^{n-1} \times[a, b] \rightarrow \mathcal{M}$ absolute $(\varepsilon, k)$-cylindrical if it satisfies the following two inequalities.

1.

$$
\left|\frac{1}{r^{2}\left(\frac{a+b}{2}\right)} \cdot g-\bar{g}\right|_{\bar{g}} \leq \varepsilon \text { on } S^{n-1} \times[a, b]
$$

where $r\left(\frac{a+b}{2}\right)$ is the mean radius of $S^{n-1} \times\left\{\frac{a+b}{2}\right\}$.

2 .

$$
\left|\bar{\nabla}^{j}\left(\frac{1}{r^{2}\left(\frac{a+b}{2}\right)} \cdot g\right)\right|_{\bar{g}} \leq \varepsilon \text { on } S^{n-1} \times[a, b]
$$

for all $1 \leq j \leq k$, where $\bar{\nabla}$ is the covariant derivative with respect to $\bar{g}$.

We call a topological neck an absolute $(\varepsilon, k, L)$-neck if it is a normal absolute $(\varepsilon, k)$-cylindrical neck and $b-a \geq 2 L$. When $\left(\varepsilon_{1}, k_{1}, L_{1}\right) \preccurlyeq\left(\varepsilon_{2}, k_{2}, L_{2}\right)$, an absolute $\left(\varepsilon_{1}, k_{1}, L_{1}\right)$-neck is an absolute $\left(\varepsilon_{2}, k_{2}, L_{2}\right)$-neck. The following result, whose proof is given in Appendix A, holds for necks in arbitrary Riemannian manifolds.

Lemma 8. Given $(\varepsilon, k, L)$, there exists $\varepsilon^{\prime}=\varepsilon^{\prime}(\varepsilon, k, L) \leq \varepsilon$ such that if $N: S^{n-1} \times[a, b] \rightarrow \mathcal{M}$ is an $\left(\varepsilon^{\prime}, k, L\right)$-neck, then $N$ is an absolute $(\varepsilon, k, L)$ neck.

Let $N: S^{n-1} \times[-L, L] \rightarrow \mathcal{M}^{n}$ be an embedded neck in a complete noncompact Riemannian manifold $\left(\mathcal{M}^{n}, g\right)$ with positive sectional curvature. By Gromoll and Meyer [GM-69], $\mathcal{M}^{n}$ is diffeomorphic to $\mathbb{R}^{n}$. Thus it follows from the solution of the Schoenflies Conjecture in dimension $n \neq 4$ [M-59], [B-60] that the center sphere $N\left(S^{n-1} \times\{0\}\right)$ bounds a differentiable ball in 
$\mathcal{M}^{n}$ when $n \neq 4$. When $n=4$ Hamilton proved the following lemma (see Theorem 1.1 in $\S 7$ of [H-97], the proof there works for all $n \geq 2$ ).

Lemma 9. There exists $\left(\varepsilon_{a}, k_{a}, L_{a}\right)$ having the following property. For any $(\varepsilon, k, L) \preccurlyeq\left(\varepsilon_{a}, k_{a}, L_{a}\right)$ and any complete noncompact Riemannian manifold $\left(\mathcal{M}^{n}, g\right)$ with positive sectional curvature which has an embedded $(\varepsilon, k, L)$ neck $N: S^{n-1} \times[-L, L] \rightarrow \mathcal{M}^{n}$, the center sphere $N\left(S^{n-1} \times\{0\}\right)$ bounds a differentiable ball in $\mathcal{M}^{n}$.

\subsection{Necks and relative volumes.}

Let $\omega_{n-1}$ be the volume of the sphere $S^{n-1}$ of radius 1 , and $\varepsilon_{b} \doteqdot \varepsilon^{\prime}\left(\frac{1}{10}, 1, L_{b}\right)$ be a function of $L_{b}$ as in Lemma 8 .

Proposition 10. For any $\delta>0$ there is $L_{b} \geq \max \left(L_{a}, 16\right)$ having the following property. For any $(\varepsilon, k, L) \preccurlyeq\left(\min \left(\varepsilon_{a}, \varepsilon_{b}\right), k_{a}, L_{b}\right)$, if there is an embedded $(\varepsilon, k, L)$-neck $N$ in a complete noncompact Riemannian manifold $\left(\mathcal{M}^{n}, g\right)$ with positive sectional curvature

$$
N: S^{n-1} \times[-L, L] \rightarrow\left(\mathcal{M}^{n}, g\right),
$$

then without any loss of generality we may assume that the component of $\mathcal{M}-N\left(S^{n-1} \times\left[-L_{b}, L_{b}\right]\right)$ bounded by $N\left(S^{n-1} \times\left\{-L_{b}\right\}\right)$ is diffeomorphic to a ball and for any $Q \in N\left(S^{n-1} \times\left\{-L_{b}\right\}\right)$ and there exists $r_{0}>0$ such that for any $R_{2} \geq R_{1} \geq r_{0}$ the following relative volume estimate holds:

$$
\frac{\operatorname{Vol}\left[\bar{B}\left(Q, R_{2}\right) \backslash B\left(Q, R_{1}\right)\right]}{\frac{\omega_{n-1}}{n} \cdot\left(R_{2}^{n}-R_{1}^{n}\right)} \leq \delta .
$$

Remark 11. The $r_{0}>0$ we shall choose has the property that for all $R_{2} \geq$ $R_{1} \geq r_{0}, \bar{B}\left(Q, R_{2}\right) \backslash B\left(Q, R_{1}\right)$ is contained in the component (diffeomorphic to $\left.S^{n-1} \times \mathbb{R}\right)$ of $\mathcal{M}-N\left(S^{n-1} \times\left[-L_{b}, L_{b}\right]\right)$ bounded by $N\left(S^{n-1} \times\left\{L_{b}\right\}\right)$.

Recall that a geodesic is called normal if it is parametrized by arc length. To prove this proposition we need the following form of the relative volume comparison theorem (see Theorem 3.1, p. 226 in Zhu [Z-97]). Let $\Gamma$ be any measurable subset of the unit sphere $S_{p}^{n-1} \subset T_{p} \mathcal{M}$. Given $r \leq R$, let

$$
A_{r, R}^{\Gamma}(p) \doteqdot\left\{\begin{array}{cc} 
& r \leq d(x, p) \leq R \text { and there exists a } \\
x \in \mathcal{M}: \quad \text { normal minimal geodesic } \gamma \text { from } \\
\gamma(0)=p \text { to } x \text { satisfying } \gamma^{\prime}(0) \in \Gamma
\end{array}\right\}
$$


Fix any point $p_{H}$ in the simply-connected space form of dimension $n$ and of constant sectional curvature $H$, let $A_{r, R}^{\Gamma}\left(p_{H}\right)$ be the corresponding set in the space form. Clearly $A_{r, R}^{\Gamma}(p) \subset \bar{B}(p, R) \backslash B(p, r)$, and if $\Gamma=S_{p}^{n-1}$ then $A_{r, R}^{\Gamma}(p)=\bar{B}(p, R) \backslash B(p, r)$.

Lemma 12. (Bishop-Gromov relative volume comparison theorem) Let $\left(\mathcal{M}^{n}, g\right)$ be a complete Riemannian manifold with Ric $_{\mathcal{M}} \geq(n-1) H$. If $r \leq R, s \leq S, r \leq s, R \leq S$ and $\Gamma$ is as above, then

$$
\frac{\operatorname{Vol}\left(A_{s, S}^{\Gamma}(p)\right)}{\operatorname{Vol}^{H}\left(A_{s, S}^{\Gamma}\left(p_{H}\right)\right)} \leq \frac{\operatorname{Vol}\left(A_{r, R}^{\Gamma}(p)\right)}{\operatorname{Vol}^{H}\left(A_{r, R}^{\Gamma}\left(p_{H}\right)\right)},
$$

where $\mathrm{Vol}^{H}$ is the volume in the space form.

Proof of Proposition 10. By multiplying the metric $g$ by a positive constant if necessary, we may assume that the center sphere $N\left(S^{n-1} \times\{0\}\right)$ has mean radius $r(0)=1$. Note that $N$ remains an $(\varepsilon, k, L)$-neck after the scaling and the desired estimate (5) does not change after the scaling. Let $N^{b}=\left.N\right|_{S^{n-1} \times\left[-L_{b}, L_{b}\right]}: S^{n-1} \times\left[-L_{b}, L_{b}\right] \rightarrow \mathcal{M}$. It is clear that $N^{b}$ is a $\left(\varepsilon, k, L_{b}\right)$-neck.

It follows from Lemma 9 that $\mathcal{M}-N^{b}\left(S^{n-1} \times\left[-L_{b}, L_{b}\right]\right)$ has two components $\mathcal{U}_{1}$ and $\mathcal{U}_{2}$, where $\mathcal{U}_{1}$ is diffeomorphic to an open ball $B^{n}$ and without loss of generality we may assume bounds $N^{b}\left(S^{n-1} \times\left\{-L_{b}\right\}\right)$, and $\mathcal{U}_{2}$ is diffeomorphic to $S^{n-1} \times \mathbb{R}$ and bounds $N^{b}\left(S^{n-1} \times\left\{L_{b}\right\}\right)$. Let $Q \in N^{b}\left(S^{n-1} \times\left\{-L_{b}\right\}\right)$. Define for $R_{2}>R_{1}>0$

$$
\Gamma=\left\{\gamma^{\prime}(0) \in S_{Q}^{n-1}: \begin{array}{c}
\text { there is } x \text { such that } R_{1} \leq d(Q, x) \leq R_{2} \\
\text { and there is exactly one normal minimal } \\
\text { geodesic } \gamma \text { from } \gamma(0)=Q \text { to } x .
\end{array}\right\} .
$$

Then $A_{R_{1}, R_{2}}^{\Gamma}(Q)$ is a subset of $\bar{B}\left(Q, R_{2}\right) \backslash B\left(Q, R_{1}\right)$ and

$$
\operatorname{Vol}\left[A_{R_{1}, R_{2}}^{\Gamma}(Q)\right]=\operatorname{Vol}\left[\bar{B}\left(Q, R_{2}\right) \backslash B\left(Q, R_{1}\right)\right]
$$

since $\left[\bar{B}\left(Q, R_{2}\right) \backslash B\left(Q, R_{1}\right)\right] \backslash A_{R_{1}, R_{2}}^{\Gamma}(Q)$ is contained in the set of cut locus points of $Q$, which has measure zero.

Let $H=0$. The corresponding space form is Euclidean space, and

$$
\operatorname{Vol}^{0}\left(A_{R_{1}, R_{2}}^{\Gamma}(0)\right)=\frac{m(\Gamma)}{n} \cdot\left(R_{2}^{n}-R_{1}^{n}\right)
$$

where $m(\Gamma)$ is the measure of $\Gamma$ in $S_{Q}^{n-1}$. We will apply Lemma 12 to (6) to prove (5). Thus we need to find for comparison another relative volume 
which is less or equal to $\delta$. We will find this other relative volume by using the embedded neck.

Choose $r_{0}$ large enough (depending on the neck $N^{b}$ and the manifold $\mathcal{M})$, so that $\left.B\left(Q, r_{0}\right) \supset \mathcal{U}_{1} \cup N^{b}\left(S^{n-1} \times\left[-L_{b}, L_{b}\right]\right]\right)$. This implies that if $R_{2}>R_{1}>r_{0}$, then $A_{R_{1}, R_{2}}^{\Gamma}(Q) \subset \mathcal{U}_{2}$.

For any normal minimal geodesic $\gamma_{0}:\left[0, \ell_{0}\right] \rightarrow \mathcal{M}$ with $\gamma_{0}(0)=Q$, $\gamma_{0}\left(\ell_{0}\right) \in A_{R_{1}, R_{2}}^{\Gamma}(Q)$ and $\gamma_{0}^{\prime}(0) \in \Gamma$, then $\gamma_{0}$ will intersect $N^{b}\left(S^{n-1} \times\{0\}\right)$ at some (exactly one) point, say $\gamma_{0}\left(w_{0}\right)$, and we claim that

$$
w_{0} \geq \frac{9}{10} L_{b} .
$$

To see the claim, since $\varepsilon_{b} \doteqdot \varepsilon^{\prime}\left(\frac{1}{10}, 1, L_{b}\right)$, by Lemma $8 N^{b}$ is an absolute $\left(\frac{1}{10}, 1, L_{b}\right)$-neck and hence (here we use the assumption that mean radius $r(0)=1)$

$$
\frac{9}{10}|\cdot|_{N_{*}^{b} \bar{g}} \leq|\cdot|_{g} \leq \frac{11}{10}|\bullet|_{N_{*}^{b} \bar{g}}
$$

where $N_{*}^{b} \bar{g}$ is the push-forward metric. From $\gamma_{0}\left(w_{0}\right) \in N^{b}\left(S^{n-1} \times\{0\}\right)$ and $Q=\gamma_{0}(0) \in N^{b}\left(S^{n-1} \times\left\{-L_{b}\right\}\right)$ we have

$$
d_{N_{*} \bar{g}}\left(\gamma_{0}(0), \gamma_{0}\left(w_{0}\right)\right) \geq L_{b} .
$$

Hence

$$
w_{0}=d_{g}\left(\gamma_{0}(0), \gamma_{0}\left(w_{0}\right)\right) \geq \frac{9}{10} d_{N_{*} \bar{g}}\left(\gamma_{0}(0), \gamma_{0}\left(w_{0}\right)\right)=\frac{9}{10} L_{b} .
$$

We now claim that (this is only a rough estimate)

$$
w_{0}+10 \leq r_{0} .
$$

Since $\mathcal{M} \backslash B\left(Q, r_{0}\right) \subset \mathcal{U}_{2}$, we have

$$
r_{0} \geq d_{g}\left(Q, \mathcal{U}_{2}\right) \geq d_{g}\left(Q, N^{b}\left(S^{n-1} \times\left\{L_{b}\right\}\right) .\right.
$$

Since any minimal geodesic from $Q$ to any $p \in N^{b}\left(S^{n-1} \times\left\{L_{b}\right\}\right)$ must intersect $N^{b}\left(S^{n-1} \times\{0\}\right)$, we have

$$
\begin{aligned}
d_{g}\left(Q, N^{b}\left(S^{n-1} \times\left\{L_{b}\right\}\right)\right. & \geq d_{g}\left(Q, N^{b}\left(S^{n-1} \times\{0\}\right)\right. \\
& +d_{g}\left(N ^ { b } \left(S^{n-1} \times\{0\}, N^{b}\left(S^{n-1} \times\left\{L_{b}\right\}\right) .\right.\right.
\end{aligned}
$$

By (8) the diameter

$$
\operatorname{diam}_{g}\left(S^{n-1} \times\{0\}\right) \leq \frac{11}{10} \cdot \operatorname{diam}_{N_{*}^{b} \bar{g}}\left(S^{n-1} \times\{0\}\right)=\frac{11 \pi}{10},
$$


it follows

$$
d_{g}\left(Q, N^{b}\left(S^{n-1} \times\{0\}\right) \geq d_{g}\left(Q, \gamma\left(w_{0}\right)\right)-\frac{11 \pi}{10}=w_{0}-\frac{11 \pi}{10} .\right.
$$

From (8) we have

$$
\begin{aligned}
& d_{g}\left(N ^ { b } \left(S^{n-1} \times\{0\}, N^{b}\left(S^{n-1} \times\left\{L_{b}\right\}\right)\right.\right. \\
& \geq \frac{9}{10} \cdot d_{N_{*}^{b} \bar{g}}\left(N ^ { b } \left(S^{n-1} \times\{0\}, N^{b}\left(S^{n-1} \times\left\{L_{b}\right\}\right)=\frac{9}{10} \cdot L_{b}\right.\right.
\end{aligned}
$$

Hence

$$
w_{0}-\frac{11 \pi}{10}+\frac{9}{10} \cdot L_{b} \leq r_{0}
$$

The claim follows since $L_{b} \geq 16$.

Now we choose the $r, R, s$ and $S$ in Lemma 12 as

$$
r \doteqdot w_{0}-2, \quad R \doteqdot w_{0}+2, \quad s \doteqdot R_{1}, \quad S \doteqdot R_{2}
$$

It is clear from the choice of $L_{b} \geq 16$ and $r_{0}<R_{1}$ that $r \leq R \leq s \leq S$. We claim

\section{Sublemma}

$$
A_{w_{0}-2, w_{0}+2}^{\Gamma}(Q) \subset\left\{x \in \mathcal{M}: d_{g}\left(x, N^{b}\left(S^{n-1} \times\{0\}\right)\right) \leq 6\right\},
$$

where $\Gamma$ is the set defined above.

Remark 13. Intuitively, the set $\left\{x \in \mathcal{M}: d_{g}\left(x, N^{b}\left(S^{n-1} \times\{0\}\right)\right) \leq 6\right\}$ is close to a standard cylinder of length 12 and radius 1 .

Problem 14. Given $R_{1}$ and $R_{2}$, let $\Gamma$ be defined as above.

Proof of sublemma. Let $R_{1}, R_{2}, \Gamma$ and $w_{0}$ be as above. Note that $\gamma_{0}\left(w_{0}\right) \in$ $N^{b}\left(S^{n-1} \times\{0\}\right)$ and $\Gamma$ corresponds to $R_{1}$ and $R_{2}$. Given any point $x \in$ $A_{w_{0}-2, w_{0}+2}^{\Gamma}(Q)$, let $\gamma:\left[0, \ell_{1}\right] \rightarrow \mathcal{M}$ be a normal minimal geodesic with $\gamma(0)=Q, \gamma^{\prime}(0) \in \Gamma, \gamma\left(\ell_{1}\right) \in \bar{B}\left(Q, R_{2}\right) \backslash B\left(Q, R_{1}\right)$ and $\gamma\left(\ell_{x}\right)=x$ for some $\ell_{x} \in\left[w_{0}-2, w_{0}+2\right]$. The geodesic $\gamma$ exists since $x \in A_{w_{0}-2, w_{0}+2}^{\Gamma}(Q)$ implies there exists a normal minimal geodesic $\bar{\gamma}:\left[0, \ell_{x}\right] \rightarrow \mathcal{M}$ with $\bar{\gamma}^{\prime}(0) \in \Gamma$, $\bar{\gamma}\left(\ell_{x}\right)=x$ and $\ell_{x} \in\left[w_{0}-2, w_{0}+2\right]$. Since $\bar{\gamma}^{\prime}(0) \in \Gamma, \bar{\gamma}$ extends to a normal minimal geodesic $\gamma$ as above. From $\gamma\left(\ell_{1}\right) \notin B\left(Q, R_{1}\right)$, we have $\ell_{1} \geq R_{1}>$ $r_{0}$. Then $\gamma\left(\ell_{1}\right) \notin \mathcal{U}_{1} \cup N^{b}\left(S^{n-1} \times\left[-L_{b}, L_{b}\right]\right)$ and hence $\gamma$ will intersect 
$N^{b}\left(S^{n-1} \times\{0\}\right)$ at some point $\gamma\left(w_{x}\right)$ (for the same reason as before as applied to $\left.\gamma_{0}\right)$. That is,

$$
\gamma\left(w_{x}\right) \in N^{b}\left(S^{n-1} \times\{0\}\right) .
$$

Since the mean radius of $N^{b}\left(S^{n-1} \times\{0\}\right)$ is 1 , we have by (8)

$$
d_{g}\left(\gamma\left(w_{x}\right), \gamma_{0}\left(w_{0}\right)\right) \leq \frac{11 \pi}{10} .
$$

Hence we get

$$
\left|w_{x}-w_{0}\right| \leq \frac{11 \pi}{10} \leq 4
$$

From the triangle inequality

$$
\left|d_{g}\left(Q, \gamma\left(w_{x}\right)\right)-d_{g}\left(Q, \gamma_{0}\left(w_{0}\right)\right)\right| \leq d_{g}\left(\gamma\left(w_{x}\right), \gamma_{0}\left(w_{0}\right)\right) .
$$

On the other hand

$$
w_{0}-2 \leq d_{g}(Q, x)=\ell_{x} \leq w_{0}+2 .
$$

Combining (10) and (11) we get

$$
\left|l_{x}-w_{x}\right| \leq 6
$$

which implies

$$
d_{g}\left(\gamma\left(w_{x}\right), x\right)=d_{g}\left(\gamma\left(w_{x}\right), \gamma\left(\ell_{x}\right)\right) \leq 6 .
$$

Since $\gamma\left(w_{x}\right) \in N\left(S^{n-1} \times\{0\}\right)$,

$$
d_{g}\left(N^{b}\left(S^{n-1} \times\{0\}\right), x\right) \leq 6 .
$$

This completes the proof of the sublemma.

It follows from the sublemma and (8) that

$$
\begin{aligned}
& \operatorname{Vol}\left[A_{w_{0}-2, w_{0}+2}^{\Gamma}(Q)\right] \\
& \leq \operatorname{Vol}\left(\left\{x \in \mathcal{M}: d_{g}\left(x, N^{b}\left(S^{n-1} \times\{0\}\right)\right) \leq 6\right\}\right) \\
& \leq \operatorname{Vol}\left(\left\{x \in \mathcal{M}: d_{N_{*}^{b} \bar{g}}\left(x, N^{b}\left(S^{n-1} \times\{0\}\right)\right) \leq \frac{10}{9} \cdot 6\right\}\right) \\
& \leq\left(\frac{11}{10}\right)^{n} \cdot \operatorname{Vol}_{N_{*}^{b} \bar{g}}\left(\left\{x \in \mathcal{M}: d_{N_{*} \bar{g}}\left(x, N^{b}\left(S^{n-1} \times\{0\}\right)\right) \leq \frac{10}{9} \cdot 6\right\}\right) \\
& =\left(\frac{11}{10}\right)^{n} \cdot \omega_{n-1} \cdot 1^{n} \cdot \frac{10}{9} \cdot 12 .
\end{aligned}
$$


We now finish the proof of the proposition. Applying Lemma 12 we get

$$
\frac{\operatorname{Vol}\left[B\left(Q, R_{2}\right) \backslash B\left(Q, R_{1}\right)\right]}{\frac{m(\Gamma)}{n} \cdot\left(R_{2}^{n}-R_{1}^{n}\right)} \leq \frac{\operatorname{Vol}\left(A_{w_{0}-2, w_{0}+2}^{\Gamma}(Q)\right)}{\frac{m(\Gamma)}{n} \cdot\left(\left(w_{0}+2\right)^{n}-\left(w_{0}-2\right)^{n}\right)}
$$

since $w_{0}-2 \leq w_{0}+2 \leq r_{0} \leq R_{1} \leq R_{2}$. Replacing the common factor $m(\Gamma)$ by $\omega_{n-1}$ and applying the estimate above, we get

$$
\begin{aligned}
\frac{\operatorname{Vol}\left[B\left(Q, R_{2}\right) \backslash B\left(Q, R_{1}\right)\right]}{\frac{\omega_{n-1}}{n} \cdot\left(R_{2}^{n}-R_{1}^{n}\right)} & \leq \frac{\operatorname{Vol}\left(A_{w_{0}-2, w_{0}+2}^{\Gamma}(Q)\right)}{\frac{\omega_{n-1}}{n} \cdot\left(\left(w_{0}+2\right)^{n}-\left(w_{0}-2\right)^{n}\right)} \\
& \leq \frac{\left(\frac{11}{10}\right)^{n} \cdot \omega_{n-1} \cdot \frac{10}{9} \cdot 12}{\frac{\omega_{n-1}}{n} \cdot\left(\left(w_{0}+2\right)^{n}-\left(w_{0}-2\right)^{n}\right)}
\end{aligned}
$$

We have proved by using (7)

$$
\frac{\operatorname{Vol}\left[B\left(Q, R_{2}\right) \backslash B\left(Q, R_{1}\right)\right]}{\frac{\omega_{n-1}}{n} \cdot\left(R_{2}^{n}-R_{1}^{n}\right)} \leq \frac{\left(\frac{11}{10}\right)^{n} \cdot \omega_{n-1} \cdot \frac{10}{9} \cdot 12}{\frac{\omega_{n-1}}{n} \cdot\left(\left(\frac{9}{10} L_{b}+2\right)^{n}-\left(\frac{9}{10} L_{b}-2\right)^{n}\right)} .
$$

If we choose $L_{b} \geq \max \left(L_{a}, 16\right)$ satisfying

$$
\frac{\left(\frac{11}{10}\right)^{n} \cdot \omega_{n-1} \cdot \frac{10}{9} \cdot 12}{\frac{\omega_{n-1}}{n} \cdot\left(\left(\frac{9}{10} L_{b}+2\right)^{n}-\left(\frac{9}{10} L_{b}-2\right)^{n}\right)} \leq \delta,
$$

and choose $\varepsilon_{b}=\varepsilon^{\prime}\left(\frac{1}{10}, 1, L_{b}\right)$, then the proposition follows from (13) and (14).

\section{Proof of the main result.}

The main part of this section is devoted to estimate the relative volume in (5) from below by ASCR when $R_{1}$ and $R_{2}$ is large and dimension $n$ is odd; see Proposition 21 below. The main result Theorem 3 is proved at the very end of this section.

\subsection{Asymptotic scalar curvature ratio.}

Let $\left(\mathcal{M}^{n}, g\right)$ be a complete, noncompact Riemannian manifold with positive sectional curvature and $Q \in \mathcal{M}$. Define a function $a: \overline{\mathbb{R}}^{+} \rightarrow \overline{\mathbb{R}}^{+}$by

$$
a(r)^{2}=\sup _{x \in \mathcal{M} \backslash B(Q, r)} R(x) d(x, Q)^{2},
$$


a function $\kappa(r): \overline{\mathbb{R}}^{+} \rightarrow \overline{\mathbb{R}}^{+}$by

$$
\kappa(r)=\sup _{x \in \mathcal{M} \backslash B(Q, r)} R(x),
$$

and a function $\rho(r): \overline{\mathbb{R}}^{+} \rightarrow \overline{\mathbb{R}}^{+}$by

$$
\rho(r) \doteqdot \frac{\pi r}{4 a(r)} .
$$

The following lemma is clear.

Lemma 15. (i) $a(r)$ is positive and monotone nonincreasing.

(ii) $\operatorname{ASCR}(g)=\lim _{r \rightarrow \infty} a(r)^{2}$.

(iii) $\kappa(r) \cdot r^{2} \leq a(r)^{2}$.

To prove Proposition 21, we assume $\operatorname{ASCR}(g)<+\infty$ since otherwise the proposition is clearly true. For any $\eta_{1} \in(0,1)$ there is $r_{1}=r_{1}\left(\eta_{1}, \mathcal{M}\right)$ such that

$$
a(r) \leq \sqrt{A S C R(g)}+\eta_{1}, \text { for all } r \geq r_{1} .
$$

Clearly we have

$$
\begin{array}{ll}
\frac{a(r)}{r} \rightarrow 0^{+} \quad \text { as } r \rightarrow+\infty & \\
\rho(r) \rightarrow+\infty \quad \text { as } r \rightarrow+\infty & \text { for } r \geq r_{1} .
\end{array}
$$

\subsection{The hypersurfaces $\widehat{S}_{r}(\rho)$.}

Let $S_{r}$ be the level set of the Busemann function $b_{Q}$ (defined in $\S 3.1$ ) and $C_{r}$ be the sublevel set of the Busemann function $b_{Q}$. If $S_{r}$ is smooth, we define $\widehat{b}_{Q} \doteqdot b_{Q}, \widehat{C}_{r} \doteqdot C_{r}$ and $\widehat{S}_{r} \doteqdot S_{r}$. If $S_{r}$ is not smooth, since $\mathcal{M}$ has positive sectional curvature so $b_{Q}$ is strictly convex [GW-74], we can smooth $b_{Q}$ (see, for example, p. 158 of [ESS-89] or [GW-76]). For any positive $\eta_{2} \leq 1$ there is a smooth and strictly convex function $\widehat{b}_{Q}$ such that

$$
\left|\widehat{b}_{Q}(x)-b_{Q}(x)\right|<\eta_{2} \text { for all } x \in \mathcal{M} \text {. }
$$

We define $\widehat{C}_{r} \doteqdot \widehat{b}_{Q}^{-1}(-\infty, r)$ and $\widehat{S}_{r} \doteqdot \widehat{b}_{Q}^{-1}(r)$. So in any case we have a smooth and strictly convex hypersurface $\widehat{S}_{r}$ and a strictly convex set $\widehat{C}_{r}$. It is clear that $\widehat{S}_{r} \doteqdot \partial \widehat{C}_{r}$. 
We define the hypersurfaces $\widehat{S}_{r}(\rho)$ parallel to $\widehat{S}_{r}$ by

$$
\widehat{S}_{r}(\rho) \doteqdot\left\{x \in \mathcal{M}: d\left(x, \widehat{C}_{r}\right)=\rho\right\}
$$

The following, which is Lemma 2 on p. 157 of [ESS-89], gives an estimate for the second fundamental form of these parallel hypersurfaces.

Lemma 16. (bounds for the 2nd fundamental form of parallel hypersurfaces) (i) If sectional curvature $K \leq \varepsilon^{2}$ on $\mathcal{M}-\widehat{C}_{r}$, then the parallel hypersurfaces are smooth embedded hypersurfaces for $0<\rho<\pi /(2 \varepsilon)$.

(ii) Let $g_{r}^{\rho}$ and $h_{r}^{\rho} \in C^{\infty}\left(S^{2} T^{*} \widehat{S}_{r}(\rho)\right)$ denote the first and second fundamental forms of $\widehat{S}_{r}(\rho)$, respectively. Then

$$
-\varepsilon \tan (\varepsilon \rho) g_{r}^{\rho} \leq h_{r}^{\rho} \leq \frac{1}{\rho} g_{r}^{\rho} .
$$

(iii) Taking $\rho=\pi /(4 \varepsilon)$ in (ii) we have

$$
-\varepsilon g_{r}^{\pi /(4 \varepsilon)} \leq h_{r}^{\pi /(4 \varepsilon)} \leq \frac{4 \varepsilon}{\pi} g_{r}^{\pi /(4 \varepsilon)} .
$$

(iv) The Weingarten map $L_{r}^{\pi /(4 \varepsilon)}: T \widehat{S}_{r}(\pi /(4 \varepsilon)) \rightarrow T \widehat{S}_{r}(\pi /(4 \varepsilon))$ satisfies

$$
\left\|L_{r}^{\pi /(4 \varepsilon)}\right\| \leq \frac{4 \varepsilon}{\pi}
$$

where $\|L\| \doteqdot \max _{|v|=1}|L(v)|$.

Remark 17. Note that if $\varepsilon$ is small, then $\pi /(2 \varepsilon)$ is large. That is, the parallel hypersurfaces $\widehat{S}_{r}(\rho)$ are smooth for large $\rho$. Our conclusion is that, assuming $K \leq \varepsilon^{2}$ on $\mathcal{M}-\widehat{C}_{r}$, the second fundamental form of $\widehat{S}_{r}(\pi /(4 \varepsilon))$ is small. The reason we have the weaker $1 / \rho$ upper bound on the second fundamental form is that $\widehat{S}_{r}$ could be close to a point (like a small sphere). In particular, the $1 / \rho$ upper bound is sharp for $\widehat{S}_{0}$ a point in euclidean space.

To apply Lemma 16 we need to estimate $\varepsilon$ in terms of $\kappa(r)$ and hence $a(r)$ in regards to the condition that the sectional curvature $K \leq \varepsilon^{2}$ on $\mathcal{M}-\widehat{C}_{r}$. We will use the following elementary result whose proof is given in Appendix B.

Lemma 18. (i) $\widehat{S}_{r} \subset b_{Q}^{-1}\left(\left(r-\eta_{2}, r+\eta_{2}\right)\right)$ and $\widehat{S}_{r} \subset \mathcal{M} \backslash \bar{B}\left(Q, r-\eta_{2}\right)$.

(ii) $B\left(Q, r-\eta_{2}\right) \subset b_{Q}^{-1}\left(\left(-\infty, r-\eta_{2}\right)\right) \subset \widehat{C}_{r} \subset b_{Q}^{-1}\left(\left(-\infty, r+\eta_{2}\right)\right)$.

(iii) $\widehat{S}_{r}(\rho) \subset \mathcal{M} \backslash \bar{B}\left(Q, r+\rho-\eta_{2}\right)$.

(iv) If $\widehat{S}_{r} \subset B(Q, \eta)$, then $\widehat{S}_{r}(\rho) \subset B(Q, \eta+\rho)$. 


\subsection{Estimate of the area of $\widehat{S}_{r}(\rho)$ by Gauss-Bonnet formula.}

Note that sectional curvatures are less or equal to scalar curvatures pointwise on a manifold with nonnegative sectional curvature, i.e., $K_{x} \leq R(x)$. By Lemma 18 (ii) and Lemma 15 (iii)

$$
\sup _{\mathcal{M} \backslash \widehat{C}_{r}} K_{x} \leq \sup _{\mathcal{M} \backslash B\left(Q, r-\eta_{2}\right)} R(x)=\kappa\left(r-\eta_{2}\right) \leq \frac{a\left(r-\eta_{2}\right)^{2}}{\left(r-\eta_{2}\right)^{2}}=\left(\frac{\pi}{4 \rho\left(r-\eta_{2}\right)}\right)^{2} .
$$

Hence by Lemma 16 (i), (ii) the hypersurface $\widehat{S}_{r}(\rho)$ is smooth for $\rho \leq \rho\left(r-\eta_{2}\right)$ and its Weingarten map $L_{r}(\rho)$ is bounded by

$$
\left\|L_{r}(\rho)\right\| \leq \frac{1}{\rho} \text { for any } \rho \leq \rho\left(r-\eta_{2}\right)
$$

Indeed, since $\tan \theta \leq \frac{1}{\theta}$ for $0<\theta \leq \pi / 4$, we have $\frac{\pi}{4 \rho\left(r-\eta_{2}\right)} \tan \left(\frac{\pi}{4 \rho\left(r-\eta_{2}\right)} \rho\right) \leq \frac{1}{\rho}$ if $\rho \leq \rho\left(r-\eta_{2}\right)$.

For the remainder of this section we assume that $n$ is odd and consider only $\rho \in\left(0, \rho\left(r-\eta_{2}\right)\right)$. Let $m \doteqdot(n-1) / 2$. We shall apply the GaussBonnet formula to the hypersurface $\widehat{S}_{r}(\rho)$ for $r \geq r_{1}$ as defined in subsection 4.1. Following [ESS-89] [GW-82], let $G_{r}(\rho)$ be the Gauss-Bonnet integrand of $\widehat{S}_{r}(\rho)$ with the induced metric. There are many instances of the formula for $G_{r}(\rho)$ in the literature (see for example p. 749 of [C-44] or p. 740 of $[\mathrm{GW}-82])$, in general $G_{r}(\rho)$ is defined by

$$
\frac{2}{\omega_{n-1}} G_{r}(\rho) d V=\frac{1}{2^{2 m} \pi^{m} m !} \sum \epsilon_{i_{1}, \ldots, i_{2 m}} \Omega_{i_{1} i_{2}} \wedge \cdots \wedge \Omega_{i_{2 m-1} i_{2 m}},
$$

where $\Omega_{i j}$ are the curvature 2 -forms of the induced metric on $\widehat{S}_{r}(\rho)$. Recall that $\omega_{n-1}=\operatorname{Vol}\left(S^{2 m}\right)=2^{2 m+1} \pi^{m} m ! /(2 m) !$.Hence

$$
G_{r}(\rho) d V=\frac{1}{(2 m) !} \sum \epsilon_{i_{1}, \ldots, i_{2 m}} \Omega_{i_{1} i_{2}} \wedge \cdots \wedge \Omega_{i_{2 m-1} i_{2 m}}
$$

By the Gauss-Bonnet formula

$$
\frac{2}{\omega_{n-1}} \int_{\widehat{S}_{r}(\rho)} G_{r}(\rho) d V_{\widehat{S}_{r}(\rho)}=\chi\left(\widehat{S}_{r}(\rho)\right)=2 .
$$

We need to estimate the Gauss-Bonnet integrand (see [GW-82] p. $740 \mathrm{ff}$ or [ESS-89] p. 160ff). Define

$$
Q_{r}(\rho) \doteqdot G_{r}(\rho)-\operatorname{det}\left[L_{r}(\rho)\right] .
$$


From Lemma 18 (iii) we have the following estimate of the sectional curvature $K_{x}$ of $\mathcal{M}$ at points in $\widehat{S}_{r}(\rho)$

$$
\sup _{x \in \widehat{S}_{r}(\rho)} K_{x} \leq \kappa\left(r+\rho-\eta_{2}\right)
$$

We estimate $Q_{r}(\rho)$ as on p. 160 of [ESS-89]. Below $c(n)$ is a constant depending only on $n$. For $r \geq 1 \geq \eta_{2}$

$$
\begin{aligned}
\left|Q_{r}(\rho)\right| & \leq c(n) \cdot \sum_{p=1}^{m} \kappa\left(r+\rho-\eta_{2}\right)^{p} \cdot\left(\left|L_{r}(\rho)\right|^{2}\right)^{m-p} \\
& \leq c(n) \cdot \sum_{p=1}^{m} \frac{a\left(r+\rho-\eta_{2}\right)^{2 p}}{\left(r+\rho-\eta_{2}\right)^{2 p}} \cdot\left(\frac{1}{\rho^{2}}\right)^{m-p} \\
& \leq c(n) \cdot \sum_{p=1}^{m} a\left(r+\rho-\eta_{2}\right)^{2 p} \cdot \frac{1}{\rho^{n-1}} .
\end{aligned}
$$

It follows from the monotonicity of $a(r)$ that for $r \geq 1$

$$
\left|Q_{r}(\rho)\right| \leq c(n) \cdot \frac{1}{\rho^{n-1}} \cdot \sum_{p=1}^{m} a\left(r-\eta_{2}\right)^{2 p} .
$$

From (18), (19) and (20) we get for $r \geq 1$

$$
\begin{aligned}
\omega_{n-1} & =\int_{\widehat{S}_{r}(\rho)} G_{r}(\rho) d V_{\widehat{S}_{r}(\rho)} \\
& \leq \int_{\widehat{S}_{r}(\rho)}\left(\left|Q_{r}(\rho)\right|+\left|\operatorname{det}\left[L_{r}(\rho)\right]\right|\right) d V_{\widehat{S}_{r}(\rho)} \\
& \leq \int_{\widehat{S}_{r}(\rho)}\left(c(n) \cdot \frac{1}{\rho^{n-1}} \cdot \sum_{p=1}^{m} a\left(r-\eta_{2}\right)^{2 p}+\frac{1}{\rho^{n-1}}\right) d V_{\widehat{S}_{r}(\rho)} .
\end{aligned}
$$

Thus we have obtained the following lower bound for the areas of the hypersurfaces

Lemma 19. There is a constant $c(n)$ depending only on $n$ such that for any $r \geq 1$ and $\rho \leq \rho\left(r-\eta_{2}\right)=\frac{\pi\left(r-\eta_{2}\right)}{4 a\left(r-\eta_{2}\right)}$

$$
\text { Area }\left[\widehat{S}_{r}(\rho)\right] \geq \frac{\omega_{n-1}}{c(n) \cdot \sum_{p=1}^{m} a\left(r-\eta_{2}\right)^{2 p}+1} \cdot \rho^{n-1} .
$$




\subsection{The final argument.}

Lemma 20. (i) For $r \geq 1$

$$
\widehat{S}_{r} \subset B\left(Q, \frac{r+\eta_{2}}{1-\theta\left(r-\eta_{2}\right)}\right) \backslash B\left(Q, r-\eta_{2}\right) .
$$

(ii) For $r \geq 1$ and $\rho \leq \rho\left(r-\eta_{2}\right)$

$$
\widehat{S}_{r}(\rho) \subset B\left(Q, \frac{r+\eta_{2}}{1-\theta\left(r-\eta_{2}\right)}+\rho\right) \backslash B\left(Q, r-\eta_{2}\right) .
$$

Proof. (i) For any $x \in \widehat{S}_{r}$ it follows from Lemma 18 (i) that $d(Q, x) \geq r-\eta_{2}$ and $b_{Q}(x) \leq r+\eta_{2}$. By Lemma 7 (ii), (i) we have for

$$
b_{Q}(x) \geq(1-\theta(d(Q, x))) \cdot d(Q, x) \geq\left(1-\theta\left(r-\eta_{2}\right)\right) \cdot d(Q, x),
$$

Hence

$$
d(Q, x) \leq \frac{r+\eta_{2}}{1-\theta\left(r-\eta_{2}\right)}
$$

(ii) This follows from (i) and Lemma 18 (iv).

By Lemma 7 (i) there is a $r_{2}=r_{2}(\mathcal{M})$ such that $\theta(r)<1 / 2$ for all $r \geq r_{2}$.

Proposition 21. Let $\left(\mathcal{M}^{n}, g\right)$ be a complete, noncompact Riemannian manifold with positive sectional curvature and $Q \in \mathcal{M}$. For any $\eta_{1}, \eta_{2}>0$ there is $r_{1}=r_{1}\left(\eta_{1}, \mathcal{M}\right)$ and $r_{2}=r_{2}(\mathcal{M})$ such that for any $r \geq \max \left\{r_{1}, r_{2}\right\}+5$

$$
\begin{aligned}
& \operatorname{Vol}\left[B\left(Q, \frac{r+\eta_{2}}{1-\theta\left(r-\eta_{2}\right)}+\rho\left(r-\eta_{2}\right)\right) \backslash B\left(Q, r-\eta_{2}\right)\right] \\
& \geq \frac{\omega_{n-1}}{c(n) \cdot \sum_{p=1}^{m}\left(\sqrt{A S C R(g)}+\eta_{1}\right)^{2 p}+1} \cdot \frac{\rho\left(r-\eta_{2}\right)^{n}}{n} .
\end{aligned}
$$

Proof. From Lemma 20 (ii)

$$
\cup_{0 \leq \rho \leq \rho\left(r-\eta_{2}\right)} \widehat{S}_{r}(\rho) \subset B\left(Q, \frac{r+\eta_{2}}{1-\theta\left(r-\eta_{2}\right)}+\rho\left(r-\eta_{2}\right)\right) \backslash B\left(Q, r-\eta_{2}\right)
$$


we get by the area estimate $(21)$

$$
\begin{aligned}
& \operatorname{Vol}\left[B\left(Q, \frac{r+\eta_{2}}{1-\theta\left(r-\eta_{2}\right)}+\rho\left(r-\eta_{2}\right)\right) \backslash B\left(Q, r-\eta_{2}\right)\right] \\
& \geq \operatorname{Vol}\left(\cup_{0 \leq \rho \leq \rho\left(r-\eta_{2}\right)} \widehat{S}_{r}(\rho)\right) \\
& \geq \int_{0}^{\rho\left(r-\eta_{2}\right)} \frac{\omega_{n-1}}{c(n) \cdot \sum_{p=1}^{m} a\left(r-\eta_{2}\right)^{2 p}+1} \cdot \rho^{n-1} d \rho \\
& =\frac{\omega_{n-1}}{c(n) \cdot \sum_{p=1}^{m} a\left(r-\eta_{2}\right)^{2 p}+1} \cdot \frac{\rho\left(r-\eta_{2}\right)^{n}}{n} .
\end{aligned}
$$

The proposition follows from $\theta\left(r-\eta_{2}\right) \leq \frac{1}{2}$ and $a\left(r-\eta_{2}\right) \leq \sqrt{A S C R(g)}+\eta_{1}$ when $r \geq r \geq \max \left\{r_{1}, r_{2}\right\}+5$.

Now we finish the proof of Theorem 3. Let $\left(\varepsilon_{0}, k_{0}, L_{0}\right)=$ $\left(\min \left(\varepsilon_{a}, \varepsilon_{b}\right), k_{a}, L_{b}\right)$ given in Proposition 10. Let $N: S^{n-1} \times[-L, L] \rightarrow \mathcal{M}$ be a $(\varepsilon, k, L)$-neck with $(\varepsilon, k, L) \preccurlyeq\left(\varepsilon_{0}, k_{0}, L_{0}\right)$ and $Q \in N\left(S^{n-1} \times\left\{-L_{0}\right\}\right)$; this will give us a constant $r_{0}$. Choose positive $\eta_{1}<1$ and $\eta_{2}<1$, this will give two constants $r_{1}$ and $r_{2}$. Choosing an $r \geq \max \left\{r_{0}, r_{1}, r_{2}\right\}+5$ and letting $R_{2} \doteqdot \frac{r+\eta_{2}}{1-\theta\left(r-\eta_{2}\right)}+\rho\left(r-\eta_{2}\right)$ and $R_{1} \doteqdot r-\eta_{2}$ in Proposition 10, we get from (13) and (22)

$$
\begin{aligned}
& \frac{\left(\frac{11}{10}\right)^{n} \cdot \omega_{n-1} \cdot \frac{10}{9} \cdot 12}{\frac{\omega_{n-1}}{n} \cdot\left(\left(\frac{9}{10} L_{0}+2\right)^{n}-\left(\frac{9}{10} L_{0}-2\right)^{n}\right)} \\
& \geq \frac{\frac{\omega_{n-1}}{c(n) \cdot \sum_{p=1}^{m} a\left(r-\eta_{2}\right)^{2 p}+1} \cdot \frac{\rho\left(r-\eta_{2}\right)^{n}}{n}}{\frac{\omega_{n-1}}{n} \cdot\left(\left[\frac{r+\eta_{2}}{1-\theta\left(r-\eta_{2}\right)}+\rho\left(r-\eta_{2}\right)\right]^{n}-\left(r-\eta_{2}\right)^{n}\right)} \\
& \geq \frac{1}{c(n) \cdot \sum_{p=1}^{m} a\left(r-\eta_{2}\right)^{2 p}+1} \cdot \frac{1}{\left(\frac{r+\eta_{2}}{\left[1-\theta\left(r-\eta_{2}\right)\right] \cdot \rho\left(r-\eta_{2}\right)}+1\right)^{n}} \\
& \geq \frac{1}{c(n) \cdot \sum_{p=1}^{m} a\left(r-\eta_{2}\right)^{2 p}+1} \cdot \frac{1}{\left(\frac{12}{\pi} a\left(r-\eta_{2}\right)+1\right)^{n}},
\end{aligned}
$$

where in the last inequality we have used $\frac{r+\eta_{2}}{\rho\left(r-\eta_{2}\right)} \leq \frac{6}{\pi} a\left(r-\eta_{2}\right)$ and $\theta\left(r-\eta_{2}\right) \leq 1 / 2$. This is because $r \geq 5$ and $\frac{r-\eta_{2}}{\rho\left(r-\eta_{2}\right)}=\frac{4}{\pi} a\left(r-\eta_{2}\right)$. Using 


$$
\begin{aligned}
& a\left(r-\eta_{2}\right) \leq \sqrt{A S C R(g)}+\eta_{1} \text { we get } \\
& \frac{\left(\frac{11}{10}\right)^{n} \cdot \omega_{n-1} \cdot 1^{n} \cdot \frac{10}{9} \cdot 12}{\frac{\omega_{n-1}}{n}\left(\left(\frac{9}{10} L_{0}+2\right)^{n}-\left(\frac{9}{10} L_{0}-2\right)^{n}\right)} \\
& \geq \frac{1}{c(n) \cdot \sum_{p=1}^{m}\left(\sqrt{A S C R(g)}+\eta_{1}\right)^{2 p}+1} \cdot \frac{1}{\left(\frac{12}{\pi}\left(\sqrt{A S C R(g)}+\eta_{1}\right)+1\right)^{n}} .
\end{aligned}
$$

Now it is clear that we can make $A S C R(g) \geq C_{0}$ if we choose $L_{0}$ large enough. Theorem 3 is proved.

\section{Existence of necklike points in ancient solutions.}

We first recall the following result of Hamilton which is based on an estimate of Hamilton (Theorem 24.4 in [H-95a]) and Ivey [I-93].

Lemma 22. If $\left(\mathcal{M}^{3}, g(t)\right), t \in(-\infty, \omega)$, is a complete ancient solution of the Ricci flow with bounded curvature, then $g(t)$ has nonnegative sectional curvature for all $t \in(-\infty, \omega)$.

Proof. See [H-95a] or for a more detailed proof [CKE].

We also note that in a lemma in $\S 19$ of [H-95a], Hamilton also proved that if $\left(\mathcal{M}^{n}, g(t)\right)$ is a non-Ricci flat ancient solution with nonnegative Ricci

curvature, then there exists a constant $c_{0}>0$ depending only on $n$ such that

$$
\liminf _{t \rightarrow-\infty}|t| \sup _{x \in \mathcal{M}} R(x, t) \geq c_{0} .
$$

(See also Lemma 19.4 of [H-95a] for a related result for Type I singularities.) In some sense this may be considered as an elementary gap-type result for ancient solutions to the Ricci flow regarding the geometry at $t=-\infty$ of the space-time manifold $\mathcal{M}^{n} \times(-\infty, \omega)$.

Following the terminology of [CKE] we shall say that $(x, t)$ is an ancient Type I-like c-essential point if

$$
|\operatorname{Rm}(x, t)| \cdot|t| \geq c>0 .
$$

We say that $(x, t)$ is a $\delta$-necklike point if there exists a unit 2-form $\theta$ at $(x, t)$ such that

$$
|\mathrm{Rm}-R(\theta \otimes \theta)| \leq \delta|\mathrm{Rm}|
$$


We have the following result due to the first named author which we believe to have first appeared in the unpublished notes [CKE]. Similar results were proved earlier by Hamilton in Theorem 24.6 of [H-95a] on the existence of necklike points in Type I singular solutions to the Ricci flow on compact 3 -manifolds on finite time intervals $[0, T$ ) (see also Theorem 3.3 in $\S 2.3$ of [H-97] for a similar result in dimension four) and Corollary 3.5 of $\S 2.3$ of [H-97] on the existence of necklike points in Type I-like ancient solutions with bounded positive isotropic curvature.

Theorem 23. Let $\left(\mathcal{M}^{3}, g(t)\right), t \in(-\infty, \omega)$, be a complete ancient solution of the Ricci flow with bounded positive sectional curvature. Suppose that

$$
\sup _{\mathcal{M} \times(-\infty, 0]}|t|^{\gamma} R(x, t)<\infty
$$

for some $\gamma>0$. Then either

1. $(\mathcal{M}, g(t))$ is isometric to a shrinking spherical space form, or

2. there exists a constant $c>0$ such that for all $\tau \in(-\infty, 0]$ and $\delta>0$, there exist $x \in \mathcal{M}$ and $t \in(-\infty, \tau)$ such that $(x, t)$ is an ancient Type I-like $c$-essential point and a $\delta$-necklike point.

Proof. We shall show that if for every $c>0$ there exist $\tau \in(-\infty, 0]$ and $\delta>0$ such that there are no ancient Type I-like $c$-essential $\delta$-necklike points before time $\tau$, then $(\mathcal{M}, g(t))$ is isometric to a shrinking spherical space form.

By the hypothesis, there exists $\gamma>0$ such that

$$
K \doteqdot \sup _{\mathcal{M} \times(-\infty, 0]}|t|^{\gamma} R(x, t)<\infty
$$

(When $\gamma=1$, this is the definition of an ancient Type I-like solution.) Since the scalar curvature of $(\mathcal{M}, g(t))$ is positive, the function

$$
G \doteqdot|t|^{\gamma \varepsilon / 2} \frac{|\stackrel{\circ}{\mathrm{Rm}}|^{2}}{R^{2-\varepsilon}}
$$

is well-defined. Since the sectional curvatures are positive, $|\stackrel{\circ}{R m}| \leq|R m| \leq$ $R$, and we have the estimates

$$
G \leq R^{\varepsilon}|t|^{\gamma \varepsilon / 2} \leq K^{\varepsilon}|t|^{-\gamma \varepsilon / 2},
$$


which show that $G$ is bounded for all times $-\infty<t \leq 0$ and satisfies

$$
\lim _{t \rightarrow-\infty} \sup _{x \in \mathcal{M}} G(x, t)=0 .
$$

If there are no ancient $c$-essential $\delta$-necklike points on the time interval $(-\infty, \tau]$, then for every $x \in \mathcal{M}$ and $t \in(-\infty, \tau)$ either

$$
|\operatorname{Rm}(x, t)| \cdot|t|<c
$$

or we have

$$
|\mathrm{Rm}-R(\theta \otimes \theta)|>\delta|\mathrm{Rm}|
$$

for every unit 2-form $\theta$ at $(x, t)$.

A straightforward computation yields that if $\phi$ is a nonnegative function and $\psi$ is a positive function, both defined on space and time, then

$$
\begin{aligned}
\left(\frac{\partial}{\partial t}-\Delta\right)\left(\frac{\phi^{\alpha}}{\psi^{\beta}}\right) & =\alpha \frac{\phi^{\alpha-1}}{\psi^{\beta}}\left(\frac{\partial}{\partial t}-\Delta\right) \phi-\beta \frac{\phi^{\alpha}}{\psi^{\beta+1}}\left(\frac{\partial}{\partial t}-\Delta\right) \psi \\
& -\alpha(\alpha-1) \frac{\phi^{\alpha-2}}{\psi^{\beta}}|\nabla \phi|^{2}-\beta(\beta+1) \frac{\phi^{\alpha}}{\psi^{\beta+2}}|\nabla \psi|^{2} \\
& +2 \alpha \beta \frac{\phi^{\alpha-1}}{\psi^{\beta+1}}\langle\nabla \phi, \nabla \psi\rangle .
\end{aligned}
$$

Taking $\phi=(-t)^{\gamma \varepsilon / 2}|\stackrel{\circ}{\mathrm{R} m}|^{2}, \psi=R, \alpha=1$, and $\beta=2-\varepsilon$, a computation yields

$$
\frac{\partial}{\partial t} G \leq \Delta G+\frac{2(1-\varepsilon)}{R}\langle\nabla G, \nabla R\rangle+2 J
$$

where

$$
J \doteqdot \frac{|t|^{\gamma \varepsilon / 2}}{R^{3-\varepsilon}}\left[\varepsilon|\stackrel{\circ}{\mathrm{Rm}}|^{2}\left(|\mathrm{Rm}|^{2}-\frac{\gamma R}{4|t|}\right)-P\right]
$$

and

$$
P \doteqdot \lambda^{2}(\mu-\nu)^{2}+\mu^{2}(\lambda-\nu)^{2}+\nu^{2}(\lambda-\mu)^{2} \geq 0
$$

(here $\lambda, \mu, \nu$ are the eigenvalues of $R m$.) Fix any $(x, t)$ with $t<\tau \leq 0$. If the first alternative (24) holds there with $c \leq \gamma / 8$, then we may estimate the term

$$
|\mathrm{Rm}|^{2}-\frac{\gamma R}{4|t|} \leq R\left(|\mathrm{Rm}|-\frac{\gamma}{4|t|}\right)<R\left(\frac{c}{|t|}-\frac{\gamma}{4|t|}\right) \leq-\frac{\gamma R}{8|t|}
$$

and hence dropping the $-P \leq 0$ term yields

$$
J \leq-\frac{\gamma \varepsilon}{8|t|} G \text {. }
$$


On the other hand if the second alternative (25) holds, then Lemma 27 in Appendix $\mathrm{C}$ implies there exists $\eta(\delta)>0$ such that $P \geq \eta|\mathrm{Rm}|^{2}|\mathrm{Rm}|^{2}$ whence it follows that taking $\varepsilon \leq \eta$ gives

$$
J \leq-\frac{\gamma \varepsilon}{4|t|} G .
$$

Thus in either case, $G$ is a subsolution of the heat equation for all times $-\infty<t<\tau$, because

$$
\frac{\partial}{\partial t} G \leq \Delta G+\frac{2(1-\varepsilon)}{R}\langle\nabla G, \nabla R\rangle-\frac{\gamma \varepsilon}{2|t|} G .
$$

By the weak maximum principle, which applies even when $\mathcal{M}$ is noncompact since the both the curvatures and $G$ are bounded, $\sup _{x \in \mathcal{M}} G(x, t)$ is a nonincreasing function of time. We may then use (23) to conclude that $G \equiv 0$, hence that $\left(\mathcal{M}^{3}, g(t)\right)$ locally isometric to a round $\mathcal{S}^{3}$. Since $\left(\mathcal{M}^{3}, g(t)\right)$ is complete, we conclude that it is compact and globally isometric to a spherical space form $\mathcal{S}^{3} / \Gamma$.

Now we shall assume that $\mathcal{M}^{3}$ is noncompact. Since $g(t)$ has positive sectional curvature, by a result of Gromoll and Meyer [GM-69], there is an injectivity radius estimate:

Proposition 24. $\mathcal{M}^{3}$ is diffeomorphic to $\mathbb{R}^{3}$ and

$$
\operatorname{inj}\left(\mathcal{M}^{3}, g(t)\right) \geq \frac{\pi}{\sqrt{K_{\text {sup }}(t)}}
$$

where $K_{\text {sup }}(t)$ is the supremum of the sectional curvatures of $g(t)$.

We may apply this estimate and a standard compactness theorem to obtain a cylinder limit solution. In particular, we have:

Theorem 25. If $\left(\mathcal{M}^{3}, g(t)\right), t \in(-\infty, \omega)$, is a complete noncompact Type I-like ancient solution of the Ricci flow with bounded positive sectional curvature on an orientable 3-manifold, then there exists a sequence of points and times $\left(x_{i}, t_{i}\right) \in \mathcal{M}^{3} \times(-\infty, \omega)$ such that the dilated and translated solutions $\left(\mathcal{M}^{3}, g_{i}(t), x_{i}\right), t \in\left(-\infty, \omega_{i}\right)$, where

$$
g_{i}(t)=R\left(x_{i}, t_{i}\right) \cdot g\left(t_{i}+\frac{t}{R\left(x_{i}, t_{i}\right)}\right)
$$

and $\omega_{i}=R\left(x_{i}, t_{i}\right)\left(T-t_{i}\right)$, limit to a solution $\left(\mathcal{M}_{\infty}^{3}, g_{\infty}(t), x_{\infty}\right), t \in$ $\left(-\infty, \omega_{\infty}\right)$, to the Ricci flow isometric to the standard shrinking cylinder $S^{2} \times \mathbb{R}$. 
Remark 26. The conclusion also holds with the additional condition on the sequence of times: $\lim _{i \rightarrow \infty} t_{i}=-\infty$.

Proof. Choose any sequence $\left\{\delta_{i}\right\}_{i \in \mathbb{N}}$ with $\lim _{i \rightarrow \infty} \delta_{i}=0$. By Theorem 23 (with $\gamma=1$,) since $\mathcal{M}$ is noncompact (and hence $(\mathcal{M}, g(t)$ ) is not isometric to a shrinking spherical space form), there exists a constant $c>0$ such that there exists points and times $\left(x_{i}, t_{i}\right)$ such that

$$
\left|\operatorname{Rm}\left(x_{i}, t_{i}\right)\right| \cdot\left|t_{i}\right| \geq c>0 \text {. }
$$

and

$$
|\mathrm{Rm}-R(\theta \otimes \theta)|\left(x_{i}, t_{i}\right) \leq \delta_{i}\left|\operatorname{Rm}\left(x_{i}, t_{i}\right)\right| .
$$

for some unit 2-forms $\theta_{i}$. By Gromoll and Meyer's injectivity radius estimate, we may apply Hamilton's Gromov-type compactness theorem for solutions of the Ricci flow $[\mathrm{H}-95 \mathrm{~b}]$ to the sequence of pointed solutions $\left(\mathcal{M}^{3}, g_{i}(t), x_{i}\right)$, $t \in\left(-\infty, \omega_{i}\right)$ where

$$
g_{i}(t)=R\left(x_{i}, t_{i}\right) \cdot g\left(t_{i}+\frac{t}{R\left(x_{i}, t_{i}\right)}\right)
$$

and $\omega_{i}=R\left(x_{i}, t_{i}\right)\left(T-t_{i}\right)$. We obtain a complete limit ancient solution to the Ricci flow $\left(\mathcal{M}_{\infty}^{3}, g_{\infty}(t), x_{\infty}\right), t \in\left(-\infty, \omega_{\infty}\right)$, on a noncompact ${ }^{1}$ orientable 3-manifold with bounded nonnegative sectional curvature and

$$
\operatorname{Rm}\left(g_{\infty}\left(x_{\infty}, 0\right)\right)=R\left(g_{\infty}\left(x_{\infty}, 0\right)\right)\left(\theta_{\infty} \otimes \theta_{\infty}\right)
$$

for some unit 2-form $\theta_{\infty}$. By the strong maximum principle we conclude that the universal covering solution $\left(\widetilde{\mathcal{M}}_{\infty}^{3}, \tilde{g}_{\infty}(t)\right), t \in\left(-\infty, \omega_{\infty}\right)$, is isometric to the product of $\mathbb{R}$ and a complete ancient Type I-like solution $\left(\mathcal{N}^{2}, h_{\infty}(t)\right)$, $t \in\left(-\infty, \omega_{\infty}\right)$, to the Ricci flow on a surface with bounded positive curvature. By Theorem 26.1 of [H-95a], $\left(\mathcal{N}^{2}, h_{\infty}(t)\right)$ is isometric to a shrinking round sphere (and in particular, $\mathcal{N}^{2}$ is compact.) Now there are only two noncompact orientable quotients of $S^{2} \times \mathbb{R}: S^{2} \times \mathbb{R}$ itself and $\mathbb{R} \tilde{\times} \mathbb{R} P^{2}$, the nontrivial $\mathbb{R}$-bundle over $\mathbb{R} P^{2}$. Topologically, $\mathbb{R} \tilde{\times} \mathbb{R} P^{2} \cong S^{2} \times[0, \infty) / \sim$ where $(x, 0) \sim(-x, 0)$, which is diffeomorphic to $\mathbb{R} P^{3}-\bar{B}^{3}$. If $\mathcal{M}_{\infty}^{3} \cong \mathbb{R} \tilde{\times} \mathbb{R} P^{2}$, then $\mathcal{M}^{3}$ admits an embedded (one-sided) $\mathbb{R} P^{2}$ (since $\mathcal{M}_{\infty}^{3}$ does). However, by the work of Gromoll and Meyer [GM-69], we know that $\mathcal{M}^{3}$ is diffeomorphic to $\mathbb{R}^{3}$. This yields a contradiction. Hence $\left(\mathcal{M}_{\infty}^{3}, g_{\infty}(t), x_{\infty}\right)$ is isometric to $\mathbb{R} \times\left(\mathcal{N}^{2}, h_{\infty}(t)\right)$, where $\left(\mathcal{N}^{2}, h_{\infty}(t)\right)$ is a shrinking round 2-sphere.

\footnotetext{
${ }^{1}$ If $\mathcal{M}_{\infty}^{3}$ were compact, then $\mathcal{M}^{3}$ would be diffeomorphic to $\mathcal{M}_{\infty}^{3}$, which contradicts the asumption that $\mathcal{M}^{3}$ is noncompact.
} 
We are now in a position to prove Theorem 4 assuming Theorem 3.

Proof of Theorem 4. Let $\left(\mathcal{M}^{3}, g(t)\right),-\infty<t<\omega$, be a complete orientable noncompact ancient Type I-like solution to the Ricci flow with bounded positive sectional curvature. By Theorem 25, there exists a sequence of points and times $\left(x_{i}, t_{i}\right) \in \mathcal{M}^{3} \times(-\infty, \omega)$ such that the dilated and translated solutions $\left(\mathcal{M}^{3}, g_{i}(t), x_{i}\right), t \in\left(-\infty, \omega_{i}\right)$, limit to the standard shrinking cylinder $S^{2} \times \mathbb{R}$. Now, by Theorem 3 , given any $A_{0}<\infty$, there exists $\left(\varepsilon_{0}, k_{0}, L_{0}\right)$ such that if $\left(\mathcal{M}^{3}, g\right)$ is a complete, noncompact Riemannian manifold with bounded positive sectional curvature and containing an $(\varepsilon, k, L)$-neck with $(\varepsilon, k, L) \preccurlyeq\left(\varepsilon_{0}, k_{0}, L_{0}\right)$, then $\operatorname{ASCR}(g) \geq A_{0}$. On the other hand, since $\left(\mathcal{M}^{3}, g_{i}(0), x_{i}\right)$ limits to a standard cylinder $S^{2} \times \mathbb{R}$, for $i$ large enough, there exists an $\left(\varepsilon_{0}, k_{0}, L_{0}\right)$-neck in $\left(\mathcal{M}^{3}, g_{i}(0), x_{i}\right)$. This implies

$$
\operatorname{ASCR}\left(g\left(t_{i}\right)\right)=\operatorname{ASCR}\left(g_{i}(0)\right) \geq A_{0} .
$$

In $\S 19$ of [H-95a] Hamilton has proved that the asymptotic scalar curvature ratio of a complete ancient solution to the Ricci flow with bounded nonnegative curvature operator (when $n=3$, this is the same as nonnegative sectional curvature) is constant in time. This implies that for all $t \in(-\infty, \omega)$ we have $\operatorname{ASCR}(g(t)) \geq A_{0}$. Since $A_{0}<\infty$ is arbitrary, we conclude that

$$
\operatorname{ASCR}(g(t)) \equiv \infty
$$

for all $t \in(-\infty, \omega)$.

\section{Appendices.}

\subsection{A.}

Proof of Lemma 8. It suffices to show that there is $\varepsilon^{\prime}$ such that if $N$ : $S^{n-1} \times[a, b] \rightarrow \mathcal{M}$ is an $\left(\varepsilon^{\prime}, k, L\right)$-neck then inequalities (3) and (4) are satisfied on $z \in\left[\frac{a+b}{2}-L, \frac{a+b}{2}+L\right]$.

Assume $\varepsilon^{\prime} \leq \min \left\{1, \frac{\ln 2}{2 L}\right\}$ so that $\left|\frac{d \ln r(z)}{d z}\right| \leq \varepsilon^{\prime}$. Using the inequality $\left|e^{x}-1\right| \leq 2|x|$ for $|x| \leq \ln 2$, we get for $z \in\left[\frac{a+b}{2}-L, \frac{a+b}{2}+L\right]$,

$$
\left|\ln \frac{r^{2}(z)}{r^{2}\left(\frac{a+b}{2}\right)}\right| \leq 2\left|\int_{(a+b) / 2}^{z} \frac{d}{d \zeta}(\ln r(\zeta)) d \zeta\right| \leq 2 L \varepsilon^{\prime} \leq \ln 2,
$$


which implies

$$
\left|\frac{r^{2}(z)}{r^{2}\left(\frac{a+b}{2}\right)}-1\right| \leq 2\left|\ln \frac{r^{2}(z)}{r^{2}\left(\frac{a+b}{2}\right)}\right| \leq 4 \varepsilon^{\prime} L .
$$

From $\left|\frac{1}{r^{2}(z)} g-\bar{g}\right|_{\bar{g}} \leq \varepsilon^{\prime}$ we get

$$
\left|\frac{1}{r^{2}(z)} \cdot g\right|_{\bar{g}} \leq \sqrt{n}+\varepsilon^{\prime}
$$

since $|\bar{g}|_{\bar{g}}^{2}=\bar{g}^{i j} \bar{g}_{i j}=n$. Hence for $z \in\left[\frac{a+b}{2}-L, \frac{a+b}{2}+L\right]$

$$
\begin{aligned}
\left|\frac{1}{r^{2}\left(\frac{a+b}{2}\right)} \cdot g-\bar{g}\right|_{\bar{g}} & \leq\left|\frac{1}{r^{2}(z)} \cdot g-\bar{g}\right|_{\bar{g}}+\left|\left(\frac{r^{2}(z)}{r^{2}\left(\frac{a+b}{2}\right)}-1\right) \cdot\left(\frac{1}{r^{2}(z)} \cdot g\right)\right|_{\bar{g}} \\
& \leq \varepsilon^{\prime}+4 \varepsilon^{\prime} L \cdot\left(\sqrt{n}+\varepsilon^{\prime}\right) .
\end{aligned}
$$

If we choose

$$
\varepsilon^{\prime} \leq \min \left\{1, \frac{\ln 2}{2 L}, \frac{\varepsilon}{1+8 L \sqrt{n}}\right\}
$$

inequality (3) will hold for $z \in\left[\frac{a+b}{2}-L, \frac{a+b}{2}+L\right]$.

Let

$$
R(z)=\ln \frac{r^{2}(z)}{r^{2}\left(\frac{a+b}{2}\right)} .
$$

Assume now $\varepsilon^{\prime} \leq \min \left\{\frac{1}{2}, \frac{\ln 2}{2 L}\right\}$. From $\left|\frac{d^{j} \log r(z)}{d z^{j}}\right| \leq \varepsilon^{\prime}$ for $1 \leq j \leq k$ and $e^{R(z)} \leq 1+4 \varepsilon^{\prime} L$ we get for $1 \leq j \leq k$ and $z \in\left[\frac{a+b}{2}-L, \frac{a+b}{2}+L\right]$

$$
\left|\frac{d^{j} R(z)}{d z^{j}}\right| \leq 2 \varepsilon^{\prime}
$$

and

$$
\begin{aligned}
\left|\frac{d^{j}}{d z^{j}} \frac{r^{2}(z)}{r^{2}\left(\frac{a+b}{2}\right)}\right| & =\left|\frac{d^{j}}{d z^{j}} e^{R(z)}\right| \leq c(j) \cdot e^{R(z)} \cdot \max _{i_{1}+\cdots+i_{p}=j}\left\{\left|\frac{d^{i_{1}} R(z)}{d z^{i_{1}}} \bullet \cdots \cdot \frac{d^{i_{p}} R(z)}{d z^{i_{p}}}\right|\right\} \\
& \leq c(j) \cdot\left(1+4 \varepsilon^{\prime} L\right) \cdot 2 \varepsilon^{\prime}
\end{aligned}
$$

where $c(j)$ is a constant depending only on $j$. Hence for $1 \leq j \leq k$ and 


$$
\begin{aligned}
& z \in\left[\frac{a+b}{2}-L, \frac{a+b}{2}+L\right] \\
& \left|\bar{\nabla}^{j}\left(\frac{1}{r^{2}\left(\frac{a+b}{2}\right)} \cdot g\right)\right|_{\bar{g}}=\left|\bar{\nabla}^{j}\left(e^{R(z)} \cdot \frac{1}{r^{2}(z)} \cdot g\right)\right|_{\bar{g}} \\
& \leq \sum_{i=0}^{j}\left(\begin{array}{c}
j \\
i
\end{array}\right) \cdot\left|\frac{d^{i}}{d z^{i}} e^{R(z)}\right| \cdot\left|\bar{\nabla}^{j-i}\left(\frac{1}{r^{2}(z)} \cdot g\right)\right|_{\bar{g}} \\
& \leq\left(1+4 \varepsilon^{\prime} L\right) \varepsilon^{\prime}+\sum_{i=1}^{j-1}\left(\begin{array}{c}
j \\
i
\end{array}\right) \cdot c(i) \cdot\left(1+4 \varepsilon^{\prime} L\right) \cdot 2 \varepsilon^{\prime} \cdot \varepsilon^{\prime}+c(j) \cdot\left(1+4 \varepsilon^{\prime} L\right) \cdot 2 \varepsilon^{\prime} .
\end{aligned}
$$

Note that in the last inequality above we have used $\left|\bar{\nabla}^{j-i}\left(\frac{1}{r^{2}(z)} \cdot g\right)\right|_{\bar{g}} \leq \varepsilon^{\prime}$ for $1 \leq j-i \leq k$. So there is a constant $C_{k}$ depending only on $k$, such that if we choose

$$
\varepsilon^{\prime} \leq \min \left\{\frac{1}{2}, \frac{\ln 2}{2 L}, \frac{\varepsilon}{C_{k} \cdot(1+4 L)}\right\},
$$

then inequality (4) will hold for $z \in\left[\frac{a+b}{2}-L, \frac{a+b}{2}+L\right]$. The lemma is proved.

\subsection{B.}

Proof of Lemma 18. We shall use the properties of Busemann functions in Lemma 6.

(i) For any $x \in \widehat{S}_{r}, \widehat{b}_{Q}(x)=r$. It follows from (17) that $\left|b_{Q}(x)-r\right|<\eta_{2}$. Hence $\widehat{S}_{r} \subset b_{Q}^{-1}\left(\left(r-\eta_{2}, r+\eta_{2}\right)\right)$.

For any $x \in \widehat{S}_{r}, b_{Q}(x)>r-\eta_{2}$. Since $b_{Q}(x) \leq d(Q, x), d(Q, x)>r-\eta_{2}$ and $\widehat{S}_{r} \subset \mathcal{M} \backslash \bar{B}\left(Q, r-\eta_{2}\right)$.

(ii) For any $x \in B\left(Q, r-\eta_{2}\right), d(Q, x)<r-\eta_{2}$. Since $b_{Q}(x) \leq d(Q, x)$, $b_{Q}(x)<r-\eta_{2}$ and $B\left(Q, r-\eta_{2}\right) \subset b_{Q}^{-1}\left(\left(-\infty, r-\eta_{2}\right)\right)$.

For any $x \in b_{Q}^{-1}\left(\left(-\infty, r-\eta_{2}\right)\right), b_{Q}(x)<r-\eta_{2}$. By $(17), \widehat{b}_{Q}(x)<$ $b_{Q}(x)+\eta_{2}<r$. Hence $b_{Q}^{-1}\left(\left(-\infty, r-\eta_{2}\right)\right) \subset \widehat{C}_{r}$.

For any $x \in \widehat{C}_{r}, \widehat{b}_{Q}(x)<r$. By $(17), b_{Q}(x)<\widehat{b}_{Q}(x)+\eta_{2}<r+\eta_{2}$. Hence $\widehat{C}_{r} \subset b_{Q}^{-1}\left(\left(-\infty, r+\eta_{2}\right)\right)$.

(iii) For any $x \in \widehat{S}_{r}(\rho), d\left(x, \widehat{S}_{r}\right)=d\left(x, \widehat{C}_{r}\right)=\rho$. Let $\gamma$ be a minimal geodesic from $Q$ to $x$. It is clear that $\gamma$ intersects $\widehat{S}_{r}$ at some point $\gamma(l)$, thus

$$
d(Q, x)=d(Q, \gamma(l))+d(\gamma(l), x) \geq d\left(Q, \widehat{S}_{r}\right)+d\left(\widehat{S}_{r}, x\right)>r-\eta_{2}+\rho,
$$


and hence $\widehat{S}_{r}(\rho) \subset \mathcal{M} \backslash \bar{B}\left(Q, r+\rho-\eta_{2}\right)$.

(iv) For any $x \in \widehat{S}_{r}(\rho)$ let $y \in \widehat{S}_{r}$ be a point satisfying $d(x, y)=$ $d\left(x, \widehat{S}_{r}\right)=\rho$. Then from $\widehat{S}_{r} \subset B(Q, \eta)$

$$
d(Q, x) \leq d(Q, y)+d(y, x)<\eta+\rho,
$$

so that $\widehat{S}_{r}(\rho) \subset B(Q, \eta+\rho)$.

\subsection{C.}

Here we give the proof of an estimate of Hamilton used in the proof of Theorem $23 .^{2}$

Lemma 27. If for some $\delta \in(0,1)$ we have $|\mathrm{Rm}-R(\theta \otimes \theta)|^{2} \geq \delta|\mathrm{Rm}|^{2}$ for every unit 2 -form $\theta$, then

$$
P \geq \frac{\delta}{96(3-\delta)}|\mathrm{Rm}|^{2}|\stackrel{\circ}{\mathrm{Rm}}|^{2} .
$$

Proof. We may assume without loss of generality that $|\lambda| \geq|\mu| \geq|\nu|$. The hypothesis implies that

$$
\mu^{2}+\nu^{2}+\mu \nu \geq \frac{\delta}{2}\left(\lambda^{2}+\mu^{2}+\nu^{2}\right)
$$

and hence that $\mu^{2}+\nu^{2} \geq \frac{\delta}{3-\delta} \lambda^{2}$. Since $|\mu| \geq|\nu|$ by assumption, we have

$$
\begin{aligned}
P & =\lambda^{2}(\mu-\nu)^{2}+\mu^{2}(\lambda-\nu)^{2}+\nu^{2}(\lambda-\mu)^{2} \\
& \geq \lambda^{2}(\mu-\nu)^{2}+\frac{\delta}{2(3-\delta)} \lambda^{2}(\lambda-\nu)^{2}+\nu^{2}(\lambda-\mu)^{2} .
\end{aligned}
$$

Now notice that

$$
|\mathrm{Rm}|^{2}=\lambda^{2}+\mu^{2}+\nu^{2} \leq 3 \lambda^{2}
$$

and

$$
|\stackrel{\circ}{\operatorname{Rm}}|^{2}=\frac{1}{3}\left[(\lambda-\mu)^{2}+(\lambda-\nu)^{2}+(\mu-\nu)^{2}\right] \leq \frac{4}{3}\left(\lambda^{2}+\mu^{2}+\nu^{2}\right) \leq 4 \lambda^{2} .
$$

So if $|\nu| \leq|\lambda| / 2$, we have

$$
P \geq \frac{\delta}{2(3-\delta)} \lambda^{2}(\lambda-\nu)^{2} \geq \frac{\delta}{8(3-\delta)} \lambda^{4} \geq \frac{\delta}{96(3-\delta)}|\mathrm{Rm}|^{2}|\stackrel{\circ}{\mathrm{Rm}}|^{2},
$$

\footnotetext{
${ }^{2}$ This is a version of an estimate in the proof of Theorem 24.6 of [H-95a].
} 
while if $|\nu|>|\lambda| / 2$, we get

$$
\begin{aligned}
P & >\lambda^{2}(\mu-\nu)^{2}+\frac{\delta}{2(3-\delta)} \lambda^{2}(\lambda-\nu)^{2}+\frac{1}{4} \lambda^{2}(\lambda-\mu)^{2} \\
& \geq \frac{\delta}{2(3-\delta)} \lambda^{2}\left[(\mu-\nu)^{2}+(\lambda-\nu)^{2}+(\lambda-\mu)^{2}\right] \geq \frac{\delta}{2(3-\delta)}|\mathrm{Rm}|^{2}|\stackrel{\circ}{\mathrm{Rm}}|^{2},
\end{aligned}
$$

because $\frac{1}{4}>\frac{\delta}{2(3-\delta)}$.

\section{References.}

[B-60] M. Brown, A proof of the generalized Schoenflies theorem, Bull. Amer. Math. Soc. 66 (1960) 74-76.

[CG-72] J. Cheeger and D. Gromoll, On the structure of complete manifolds of nonnegative curvature, Ann. of Math. (2) 96 (1972) 413-443.

[C-44] S.-S. Chern, A simple intrinsic proof of the Gauss-Bonnet formula for closed Riemannian manifolds, Ann. of Math. 45 (1944) 747752.

[CKE] B. Chow and D. Knopf, The Ricci flow, Volume I: an Introduction, to appear in the Mathematical Surveys and Monographs series, AMS (2004).

[D-94] G. Drees, Asymptotically flat manifolds of nonnegative curvature, Diff. Geom. Appl. 4 (1994) 77-90.

[ESS-89] J. H. Eschenburg, V. Schroeder and M. Strake, Curvature at infinity of open nonnegatively curved manifolds, J. Diff. Geom. 30 (1989) 155-166.

[G-97] R. E. Greene, "A genealogy of noncompact manifolds of nonnegative curvature: history and logic," in Comparison Geometry, ed. Grove and Petersen, MSRI Publ. 30 (1997) 99-134.

[GW-74] R. E. Greene and H. Wu, Integrals of subharmonic functions on manifolds of nonnegative curvature, Invent. Math. 27 (1974) 265298.

[GW-76] R. E. Greene and $\mathrm{H}$. Wu, $C^{\infty}$-convex functions and manifolds of positive curvature, Acta Math. 137 (1976) 209-245. 
[GW-82] R. E. Greene and H. Wu, Gap theorems for noncompact Riemannian manifolds, Duke. Math. J. 49 (1982) 731-756.

[GM-69] D. Gromoll and W. Meyer, On complete manifolds of positive curvature, Ann. of Math. 90 (1969) 75-90.

[H-93a] R. S. Hamilton, The Harnack estimate for the Ricci flow, J. Diff. Geom. 37 (1993) 225-243.

[H-93b] R. S. Hamilton, Eternal solutions to the Ricci flow, J. Diff. Geom. 38 (1993) 1-11.

[H-95a] R. S. Hamilton, "The formation of singularities in the Ricci flow," in Surveys in Differential Geometry 2 (1995) International Press, $7-136$.

[H-95b] R. S. Hamilton, A compactness property for solutions of the Ricci flow, Amer. J. Math. 117 (1995) 545-572.

[H-97] R. S. Hamilton, Four-manifolds with positive isotropic curvature, Comm. Anal. Geom. 5 (1997) 1-92.

[Hu-90] G. Huisken, Asymptotic behavior for singularities of the mean curvature flow, J. Diff. Geom. 31 (1990) 285-299.

[I-93] T. Ivey, Ricci solitons on compact 3-manifolds, Differential Geom. Appl. 3 (1993) 301-307.

[K-88] A. Kasue, A compactification of a manifold with asymptotically nonnegative curvature, Ann. Sci. Ec. Norm. Sup. IV Ser. 21 (1988) 593-622.

[KS-87] A. Kasue and K. Sugahara, Gap theorems for certain submanifolds of Euclidean spaces and hyperbolic space forms, Osaka J. Math. 24 (1987) 679-704.

[LT-87] P. Li and L.-F. Tam, Positive harmonic functions on complete manifolds with nonnegative curvature outside a compact set, Ann. of Math. 125 (1987) 171-207.

[M-59] B. Mazur, On embeddings of spheres, Bull. Amer. Math. Soc. 65 (1959) 59-65.

[PT-01] A. Petrunin and W. Tuschmann, Asymptotical flatness and cone structure at infinity, Math. Ann. 321 (2001) 775-788. 
[Z-97] S. Zhu, "The comparison geometry of Ricci curvature," in Comparison Geometry, ed. Grove and Petersen, MSRI Publ. 30 (1997) 221-262.

BennetT Chow

University of California, San Diego

PENG LU

UNIVERSITY OF OREGON 
B. Chow and P. Lu 